\title{
A free-boundary problem in combustion theory
}

\author{
JULIÁN FERNÁNDEZ BONDER ${ }^{\dagger}$ AND NOEMI WOLANSKI \\ Departamento de Matemática, FCEyN, UBA (1428) Buenos Aires, Argentina
}

[Received 21 May 1999 and in revised form 28 December 1999]

In this paper we consider the following problem arising in combustion theory:

$$
\begin{cases}\Delta u^{\varepsilon}-u_{t}^{\varepsilon}=v^{\varepsilon} f_{\varepsilon}\left(u^{\varepsilon}\right) & \text { in } \quad \mathcal{D}, \\ \Delta v^{\varepsilon}-v_{t}^{\varepsilon}=v^{\varepsilon} f_{\varepsilon}\left(u^{\varepsilon}\right) & \text { in } \quad \mathcal{D},\end{cases}
$$

where $\mathcal{D} \subset \mathbb{R}^{N+1}, f_{\varepsilon}(s)=\frac{1}{\varepsilon^{2}} f\left(\frac{s}{\varepsilon}\right)$ with $f$ a Lipschitz continuous function with support in $(-\infty, 1]$.

Here $v^{\varepsilon}$ is the mass fraction of some reactant, $u^{\varepsilon}$ the rescaled temperature of the mixture and $\varepsilon$ is essentially the inverse of the activation energy. This model is derived in the framework of the theory of equi-diffusional premixed flames for Lewis number 1 .

We prove that, under suitable assumptions on the functions $u^{\varepsilon}$ and $v^{\varepsilon}$, we can pass to the limit $(\varepsilon \rightarrow 0)$-the so-called high-activation energy limit—and that the limit function $u=\lim u^{\varepsilon}=$ $\lim v^{\varepsilon}$ is a solution of the following free-boundary problem:

$$
\begin{aligned}
& \Delta u-u_{t}=0 \quad \text { in } \quad\{u>0\} \\
& |\nabla u|=\sqrt{2 M(x, t)} \quad \text { on } \quad \partial\{u>0\}
\end{aligned}
$$

in a pointwise sense at regular free-boundary points and in a viscosity sense. Here $M(x, t)=$ $\int_{-w_{0}(x, t)}^{1}\left(s+w_{0}(x, t)\right) f(s) \mathrm{d} s$ and $-1<w_{0}=\lim _{\varepsilon \rightarrow 0} \frac{v^{\varepsilon}-u^{\varepsilon}}{\varepsilon}$.

Since $v^{\varepsilon}-u^{\varepsilon}$ is a solution of the heat equation, it is fully determined by its initial-boundary datum. In particular, the free-boundary condition only (but strongly) depends on the approximation of the initial-boundary datum.

Moreover, if $\mathcal{D} \cap \partial\{u>0\}$ is a Lipschitz surface, $u$ is a classical solution to (0.1).

\section{Introduction}

In this paper we consider the following problem arising in combustion theory:

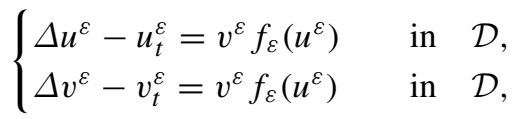

where $\mathcal{D} \subset \mathbb{R}^{N+1}$.

This model appears in combustion theory in the analysis of the propagation of curved flames. It is derived in the framework of the theory of equi-diffusional premixed flames analysed in the relevant limit of high- activation energy for Lewis number 1 . In this application, $v^{\varepsilon}$ represents the fraction of

\footnotetext{
†Email: jfbonder@dm.uba.ar
}

Email: wolanski@dm.uba.ar 
some reactant (and hence it is assumed to be non-negative), and $u^{\varepsilon}$ its temperature (more precisely, $u^{\varepsilon}=\lambda\left(T_{f}-T^{\varepsilon}\right)$ where $T_{f}$ is the flame temperature and $\lambda$ is a normalization factor). We observe that the term $v^{\varepsilon} f_{\varepsilon}\left(u^{\varepsilon}\right)$ acts as an absorption term in the equation (1.1). Since $T^{\varepsilon}=T_{f}-\left(u^{\varepsilon} / \lambda\right)$, it is in fact a reaction term for the temperature. In the flame model, such a term represents the effect of the exothermic chemical reaction and $f$ has accordingly a number of properties: it is a non-negative Lipschitz continuous function which is positive in an interval $(-\infty, \varepsilon)$ and vanishes otherwise (i.e. reaction occurs only when $T>T_{f}-\frac{\varepsilon}{\lambda}$ ). The parameter $\varepsilon$ is essentially the inverse of the activation energy of the chemical reaction. For the sake of simplicity we will assume that $f_{\varepsilon}(s)=\frac{1}{\varepsilon^{2}} f\left(\frac{s}{\varepsilon}\right)$, where $f$ is a non-negative, Lipschitz continuous function with support in $(-\infty, 1]$.

For the derivation of the model, see [3].

Here we are interested in high-activation energy limits (i.e. $\varepsilon \rightarrow 0$ ). These limits are currently the subject of active investigation, specially in the case $u^{\varepsilon}=v^{\varepsilon}$. This is a natural assumption in the case of travelling waves.

The study of the limit as $\varepsilon \rightarrow 0$ was proposed in the 1930 s by Zeldovich \& FrankKamenetski [13] and has been much discussed in the combustion literature. In the case $u^{\varepsilon}=v^{\varepsilon}$, the reaction function $u^{\varepsilon} f_{\varepsilon}\left(u^{\varepsilon}\right)$ tends to a Dirac delta, $M_{0} \delta(u)$ where $M_{0}=\int_{0}^{1} s f(s) \mathrm{d} s$. In this way the reaction zone where $u^{\varepsilon} f_{\varepsilon}\left(u^{\varepsilon}\right)$ acts is reduced to a surface, the flame front, and a free-boundary problem arises. The fact that $M_{0}>0$ ensures that a non-trivial combustion process takes place so that a non-empty free boundary actually appears.

Although the convergence of the most relevant propagation modes, i.e. the travelling waves, was already discussed by Zeldovich and Frank-Kamenetski, and an enormous progress in this direction has been made, a rigorous mathematical investigation of the convergence of general solutions is still in progress. Berestycki and his collaborators have rigorously studied the convergence problem for travelling waves and, more generally, in the elliptic stationary case; cf. [2] and its references. See also [12]. The study of the limit in the general evolution case for the heat operator has been performed in [7] for the one-phase case (this is with $u^{\varepsilon} \geqslant 0$ ) and in [4-6] for the two-phase case, where no sign restriction on $u^{\varepsilon}$ is made.

In [7] the authors show that, under certain assumptions on the initial datum and its approximations, for every sequence $\varepsilon_{n} \rightarrow 0$ there exists a subsequence $\varepsilon_{n_{k}}$ and a limit function $u=\lim u^{\varepsilon_{n_{k}}}$ which solves the following free-boundary problem:

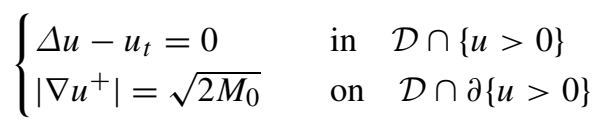

in a weak integral sense. Here $M_{0}=\int_{0}^{1} s f(s) \mathrm{d} s$.

In [5] and [6] the authors show that the free-boundary condition for the two-phase case (when it is assumed that no reaction takes place if $u^{\varepsilon} \leqslant 0$ ) is

$$
\left|\nabla u^{+}\right|^{2}-\left|\nabla u^{-}\right|^{2}=2 M_{0}
$$

and that the limit function is a solution of the free-boundary problem in a pointwise sense at regular free-boundary points when $\{u=0\}$ has zero 'parabolic density' and in a viscosity sense in the absence of a zero phase (i.e. when $\{u=0\}^{\circ} \cap \mathcal{D}=\emptyset$ ).

On the other hand, in [10] it was shown, for the one-phase problem in a cylinder with Neumann boundary conditions, that when a classical solution to the free-boundary problem (1.2) exists, it is the limit of the whole family $u^{\varepsilon}$, not just of a subsequence. Moreover, this classical solution is the 
limit of $u^{\varepsilon}$ independently of the choice of the approximate initial data $u^{\varepsilon}(x, 0)$. A similar result has been obtain for the two-phase case in [11].

So that a natural question is whether a classical solution to the free- boundary problem (1.2) is also the limit of $u^{\varepsilon}$ if $\left(u^{\varepsilon}, v^{\varepsilon}\right)$ is the solution to the system (1.1) and both $u^{\varepsilon}(x, 0)$ and $v^{\varepsilon}(x, 0)$ converge to $u(x, 0)$ but $u^{\varepsilon}(x, 0) \neq v^{\varepsilon}(x, 0)$.

Or we may ask a more elementary question: will a sequence of uniformly bounded solutions $\left(u^{\varepsilon}, v^{\varepsilon}\right)$ with $\left(v^{\varepsilon}-u^{\varepsilon}\right) \rightarrow 0$ as $\varepsilon \rightarrow 0$ be such that $u^{\varepsilon}$ converges to a solution of the free-boundary problem (1.2)? This is: will the asymptotic limit for activation energy going to infinity, in the case in which $\left(v^{\varepsilon}-u^{\varepsilon}\right) \rightarrow 0$ but $u^{\varepsilon} \neq v^{\varepsilon}$, be a solution of the same free-boundary problem as in the case in which $u^{\varepsilon}=v^{\varepsilon}$ ?

In order to understand the relation between both assertions it is important to point out that, in the case under consideration, this is when the Lewis number is 1 , the function $w^{\varepsilon}=v^{\varepsilon}-u^{\varepsilon}$ is a solution of the heat equation. So that it is fully determined by its initial-boundary datum. Moreover, the system (1.1) may be rewritten as a single equation for $u^{\varepsilon}$, namely

$$
\Delta u^{\varepsilon}-u_{t}^{\varepsilon}=\left(u^{\varepsilon}+w^{\varepsilon}\right) f_{\varepsilon}\left(u^{\varepsilon}\right) .
$$

In this paper we consider the case in which $w^{\varepsilon} / \varepsilon$ converges to a function $w_{0}$ (so that in particular, $v^{\varepsilon}-u^{\varepsilon} \rightarrow 0$ ). In this way, at least formally, the reaction term still converges to a delta function and a free- boundary problem appears. But we prove that the free-boundary condition strongly depends on the limit function $w_{0}$, so that it is different for different approximations of the initial-boundary datum of $u$.

In fact, we prove that for every sequence $\varepsilon_{n} \rightarrow 0$ there exists a subsequence $\varepsilon_{n_{k}}$ and a limit function $u=\lim u^{\varepsilon_{n_{k}}}$ which is a solution of the following free-boundary problem:

$$
\left\{\begin{array}{lll}
\Delta u-u_{t}=0 & \text { in } & \mathcal{D} \cap\{u>0\} \\
\left|\nabla u^{+}\right|=\sqrt{2 M(x, t)} & \text { on } & \mathcal{D} \cap \partial\{u>0\}
\end{array}\right.
$$

where $M(x, t)=\int_{-w_{0}(x, t)}^{1}\left(s+w_{0}(x, t)\right) f(s) \mathrm{d} s$.

The presence of the function $w_{0}$ in the limit of integration gives the necessary positive sign of the function $M(x, t)$.

In conclusion, the combustion problem is very unstable in the sense that the asymptotic limit for activation energy going to infinity depends on order $\varepsilon$ perturbations of the initial-boundary data.

In this paper we prove that the limit function $u$ is a 'viscosity' solution to (1.4), so that, as a consequence of our results and of the regularity results for viscosity solutions to (1.4) of [8], we deduce that, when the free boundary of a limit function $u$ is given by $x_{1}=g\left(x^{\prime}, t\right), x=\left(x_{1}, x^{\prime}\right)$ with $g$ Lipschitz continuous, $u$ is a classical solution.

We want to stress that because of our assumption that $v^{\varepsilon}-u^{\varepsilon} \rightarrow 0$ and since $v^{\varepsilon} \geqslant 0$, the limit function $u$ must be non-negative, so our result is new even in the case $u^{\varepsilon}=v^{\varepsilon}$.

In particular, as a consequence of our results, we see that limit functions $u$ with $u^{\varepsilon}(x, 0)$ constructed as in [7], and $v^{\varepsilon}(x, 0)$ small perturbations of $u^{\varepsilon}(x, 0)$ are viscosity solutions to (1.4). In this construction, $w_{0}$ is any constant such that $w_{0} \geqslant-\eta$ where $\eta>0$ is small enough.

Notation, hypotheses and outline of the paper. Throughout this paper $N$ will denote the spatial dimension and, in addition, the following notation will be used. 
For any $x_{0} \in \mathbb{R}^{N}, t_{0} \in \mathbb{R}$ and $\tau>0$

$$
\begin{aligned}
B_{\tau}\left(x_{0}\right) & \equiv\left\{x \in \mathbb{R}^{N} /\left|x-x_{0}\right|<\tau\right\}, \\
B_{\tau}\left(x_{0}, t_{0}\right) & \equiv\left\{(x, t) \in \mathbb{R}^{N+1} /\left|x-x_{0}\right|^{2}+\left|t-t_{0}\right|^{2}<\tau^{2}\right\}, \\
Q_{\tau}\left(x_{0}, t_{0}\right) & \equiv B_{\tau}\left(x_{0}\right) \times\left(t_{0}-\tau^{2}, t_{0}+\tau^{2}\right), \\
Q_{\tau}^{-}\left(x_{0}, t_{0}\right) & \equiv B_{\tau}\left(x_{0}\right) \times\left(t_{0}-\tau^{2}, t_{0}\right],
\end{aligned}
$$

and for any set $K \subset \mathbb{R}^{N+1}$

$$
\begin{aligned}
\mathcal{N}_{\tau}(K) & \equiv \bigcup_{\left(x_{0}, t_{0}\right) \in K} Q_{\tau}\left(x_{0}, t_{0}\right), \\
\mathcal{N}_{\tau}^{-}(K) & \equiv \bigcup_{\left(x_{0}, t_{0}\right) \in K} Q_{\tau}^{-}\left(x_{0}, t_{0}\right) .
\end{aligned}
$$

When necessary, we will denote points in $\mathbb{R}^{N}$ by $x=\left(x_{1}, x^{\prime}\right)$, with $x^{\prime} \in \mathbb{R}^{N-1}$. Also, $\langle\cdot, \cdot\rangle$ will mean the usual scalar product in $\mathbb{R}^{N}$. Given a function $v$, we will denote $v^{+}=\max (v, 0), v^{-}=$ $\max (-v, 0)$.

In addition, the symbols $\Delta$ and $\nabla$ will denote the corresponding operators in the space variables; the symbol $\partial_{p}$ will denote parabolic boundary.

Finally, we will say that a function $v$ is in the class $\operatorname{Lip}_{\text {loc }}\left(1, \frac{1}{2}\right)$ in a domain $\mathcal{D} \subset \mathbb{R}^{N+1}$, if for every $\mathcal{D}^{\prime} \subset \subset \mathcal{D}$, there exists a constant $L=L\left(\mathcal{D}^{\prime}\right)$ such that

$$
|v(x, t)-v(y, s)| \leqslant L\left(|x-y|+|t-s|^{1 / 2}\right)
$$

for every $(x, t),(y, s) \in \mathcal{D}^{\prime}$. If the constant $L$ does not depend on the set $\mathcal{D}^{\prime}$, we will say that $v \in \operatorname{Lip}\left(1, \frac{1}{2}\right)$ in $\mathcal{D}$.

For the existence of a limit function for a subsequence $u^{\varepsilon_{n_{k}}}$ we only need the weaker condition that for every compact $K \subset \mathcal{N}_{\tau}^{-}(K) \subset \mathcal{D}$,

$$
\left\|v^{\varepsilon}-u^{\varepsilon}\right\|_{L^{\infty}\left(\mathcal{N}_{\tau}^{-}(K)\right)}=\mathrm{O}(\varepsilon) .
$$

Then, we have (see [9])

$$
\left\|v^{\varepsilon}-u^{\varepsilon}\right\|_{C^{2,1}(K)}=\mathrm{O}(\varepsilon) .
$$

Under this assumption, we are able to apply the results of [4] and get the uniform Lipschitz estimates needed to pass to the limit in (1.1). This is done in Section 2 where we also prove some technical lemmas that are used throughout the paper.

In Sections 3 and 4 we assume that $u^{\varepsilon} \rightarrow 0$ in $\{u=0\}$ fast enough. This is an essential condition that was already considered in [7]. This assumption is a natural one in applications; roughly speaking it means that the mixture temperature reaches the flame temperature only if some combustion is taking place.

We also assume that there exists $\lim _{\varepsilon \rightarrow 0}\left(v^{\varepsilon}-u^{\varepsilon}\right) / \varepsilon=: w_{0}$ and, as a consequence of the hypothesis that $u^{\varepsilon} \rightarrow 0$ in $\{u=0\}$ fast enough, we show that necessarily $w_{0}>-1$ in $\{u \equiv 0\}^{\circ}$. So that, in Sections 3 and 4 we assume that for every $K \subset \mathcal{N}_{\tau}^{-}(K) \subset \mathcal{D}$ compact

$$
\frac{v^{\varepsilon}-u^{\varepsilon}}{\varepsilon} \rightarrow w_{0} \quad \text { uniformly in } \quad N_{\tau}^{-}(K) .
$$


Thus,

$$
\left\|\frac{v^{\varepsilon}-u^{\varepsilon}}{\varepsilon}-w_{0}\right\|_{C^{2,1}(K)} \rightarrow 0 .
$$

And, for the sake of simplicity, we assume that $w_{0}>-1$ in $\mathcal{D}$.

In Section 3, we show that the limit function $u$ is a solution to the free- boundary problem (1.4) in a pointwise sense.

Finally, in Section 4 we prove that the limit function $u$ is in fact a viscosity solution of the freeboundary problem (1.4) under a non-degeneracy assumption on the limit function $u$. We also prove some results that give the necessary non-degeneracy of $u$.

Our presentation is of a local nature, so that our hypotheses are stated in terms of the solution $\left(u^{\varepsilon}, v^{\varepsilon}\right)$. As can be seen in the example treated in Corollary 4.3 it is possible to deduce our hypotheses on $\left(u^{\varepsilon}, v^{\varepsilon}\right)$ from conditions on its initial-boundary datum.

\section{Uniform estimates}

In this section we consider a family $u^{\varepsilon}, v^{\varepsilon}$ of solutions to (1.1) in a domain $\mathcal{D}$ which are uniformly bounded in $L^{\infty}$ norm in $\mathcal{D}$. We show that the functions $u^{\varepsilon}, v^{\varepsilon}$ are locally, uniformly bounded in the semi-norm $\operatorname{Lip}\left(1, \frac{1}{2}\right)$. Then, we get further local uniform estimates and pass to the limit as $\varepsilon \rightarrow 0$. We also show that the limit function $u$ is a solution to the free-boundary problem (1.4) in a very weak sense. Finally, we prove an approximation lemma that will be used throughout the rest of the paper and some lemmas concerning particular limit functions. Also, we prove a proposition that justifies the hypothesis we make in the following sections.

For convenience, let us define the following function:

$$
w^{\varepsilon}(x, t)=v^{\varepsilon}(x, t)-u^{\varepsilon}(x, t),
$$

then, $w^{\varepsilon}$ is a caloric function with $\left\|w^{\varepsilon}\right\|_{C^{2,1}(K)}=\mathrm{O}(\varepsilon)$ for every compact set $K \subset \mathcal{D}$.

We begin with a proposition (which is a consequence of [4]) that gives us the uniform control on the gradients of solutions of (1.1).

Proposition 2.1 Let $\left(u^{\varepsilon}, v^{\varepsilon}\right)$ be solutions of (1.1) such that $\left\|u^{\varepsilon}\right\|_{\infty} \leqslant \mathcal{A}, v^{\varepsilon} \geqslant 0$, which verify (1.5). Let $K \subset \mathcal{D}$ be compact and $\tau>0$ such that $\mathcal{N}_{\tau}^{-}(K) \subset \mathcal{D}$. Then, there exists $L=L(\tau, \mathcal{A})$ such that

$$
\left|\nabla u^{\varepsilon}(x, t)\right| \leqslant L, \quad\left|\nabla v^{\varepsilon}(x, t)\right| \leqslant L .
$$

Proof. Let us start by making the following observation:

$$
u^{\varepsilon}=v^{\varepsilon}-w^{\varepsilon} \geqslant-w^{\varepsilon} \geqslant-C \varepsilon .
$$

Then, let $z^{\varepsilon}=\frac{1}{C+1}\left(u^{\varepsilon}+C \varepsilon\right)$ and we define, for $\left(x_{0}, t_{0}\right) \in K$,

$$
z_{\tau}^{\varepsilon}(x, t)=\frac{1}{\tau} z^{\varepsilon}\left(x_{0}+\tau x, t_{0}+\tau^{2} t\right) .
$$

In $B_{1}(0) \times[-1,0], z_{\tau}^{\varepsilon}$ verifies (with $B \geqslant\|f\|_{\infty}$ )

$$
\begin{aligned}
0 \leqslant \Delta z_{\tau}^{\varepsilon}-\frac{\partial z_{\tau}^{\varepsilon}}{\partial t} & \leqslant \frac{\tau}{C+1}\left(C \varepsilon+\left|u^{\varepsilon}\right|\right) \frac{1}{\varepsilon^{2}} f\left(\frac{u^{\varepsilon}}{\varepsilon}\right) \\
& \leqslant B \tau \frac{1}{\varepsilon} \mathcal{X}_{[-C \varepsilon, \varepsilon]}\left(u^{\varepsilon}\right)=\frac{B}{\varepsilon / \tau} \mathcal{X}_{[0, \varepsilon / \tau]}\left(z_{\tau}^{\varepsilon}\right) .
\end{aligned}
$$


On the other hand,

$$
\left|z_{\tau}^{\varepsilon}(x, t)\right| \leqslant \frac{\left|u^{\varepsilon}(x, t)\right|+C}{\tau(1+C)} \leqslant \frac{1}{\tau} \frac{\mathcal{A}+C}{1+C} .
$$

Therefore, by Theorem 2 of [4] it follows that

$$
\left|\nabla z_{\tau}^{\varepsilon}(x, t)\right| \leqslant \bar{L}=\bar{L}(\tau, \mathcal{A}) \quad \text { in } \quad B_{1 / 2}(0) \times(-1 / 2,0] .
$$

In particular,

$$
\begin{aligned}
& \left|\nabla u^{\varepsilon}\left(x_{0}, t_{0}\right)\right|=(C+1)\left|\nabla z^{\varepsilon}\left(x_{0}, t_{0}\right)\right|=(C+1)\left|\nabla z_{\tau}^{\varepsilon}(0,0)\right| \leqslant(C+1) \bar{L} \\
& \left|\nabla v^{\varepsilon}\left(x_{0}, t_{0}\right)\right| \leqslant\left|\nabla u^{\varepsilon}\left(x_{0}, t_{0}\right)\right|+\left|\nabla w^{\varepsilon}\left(x_{0}, t_{0}\right)\right| \leqslant(C+1) \bar{L}+C .
\end{aligned}
$$

The proof is complete.

As is usual in parabolic theory, Lipschitz regularity in space gives Hölder $\frac{1}{2}$ regularity in time. We follow here ideas in [5] and [7].

Proposition 2.2 Let $\left(u^{\varepsilon}, v^{\varepsilon}\right)$ be solutions of (1.1) such that $\left\|u^{\varepsilon}\right\|_{\infty} \leqslant \mathcal{A}, v^{\varepsilon} \geqslant 0$, which verify (1.5). Let $K \subset \mathcal{D}$ be compact and $\tau>0$ such that $\mathcal{N}_{\tau}(K) \subset \mathcal{D}$. Then there exists $C=C(\tau, \mathcal{A})$ such that

$$
\left|u^{\varepsilon}(x, t+\Delta t)-u^{\varepsilon}(x, t)\right| \leqslant C|\Delta t|^{1 / 2}, \quad\left|v^{\varepsilon}(x, t+\Delta t)-v^{\varepsilon}(x, t)\right| \leqslant C|\Delta t|^{1 / 2},
$$

for every $(x, t),(x, t+\Delta t) \in K$.

Proof. As in Proposition 2.1 we define $z^{\varepsilon}=\frac{1}{C+1}\left(u^{\varepsilon}+C \varepsilon\right)$ and

$$
z_{\lambda}^{\varepsilon}(x, t)=\frac{1}{\lambda} z^{\varepsilon}\left(x_{0}+\lambda x, t_{0}+\lambda^{2} t\right),
$$

for $0<\lambda<\tau$ and $\left(x_{0}, t_{0}\right) \in K$.

By a simple computation we get, as in Proposition 2.1,

$$
0 \leqslant \Delta z_{\lambda}^{\varepsilon}-\frac{\partial z_{\lambda}^{\varepsilon}}{\partial t} \leqslant \frac{B}{\varepsilon / \lambda} \mathcal{X}_{[0, \varepsilon / \lambda]}\left(z_{\lambda}^{\varepsilon}\right)
$$

Now, $z_{\lambda}^{\varepsilon} \geqslant 0$, and in $\left\{z_{\lambda}^{\varepsilon}>1\right\}$ we have

$$
\left|\Delta z_{\lambda}^{\varepsilon}-\frac{\partial z_{\lambda}^{\varepsilon}}{\partial t}\right|\left\{\begin{array}{lll}
\leqslant B & \text { if } \quad \varepsilon / \lambda \geqslant 1 \\
=0 & \text { if } \quad \varepsilon / \lambda<1
\end{array}\right.
$$

Moreover, we have that

$$
\left|\nabla z_{\lambda}^{\varepsilon}(x, t)\right|=\frac{1}{C+1}\left|\nabla u^{\varepsilon}\left(x_{0}+\lambda x, t_{0}+\lambda^{2} t\right)\right| \leqslant \bar{L}
$$

in $B_{\tau / \lambda}(0) \times\left[0, \tau^{2} / \lambda^{2}\right]$. Then, by Proposition 2.2 of [5], we have

$$
\left|z_{\lambda}^{\varepsilon}(0, t)-z_{\lambda}^{\varepsilon}(0,0)\right| \leqslant C(\bar{L}) \quad \forall 0 \leqslant t \leqslant \frac{1}{4 N+B}
$$


which, in terms of $u^{\varepsilon}$, is

$$
\left|u^{\varepsilon}\left(x_{0}, t_{0}+\lambda^{2} t\right)-u^{\varepsilon}\left(x_{0}, t_{0}\right)\right| \leqslant C(\bar{L}) \lambda .
$$

In particular,

$$
\left|u^{\varepsilon}\left(x_{0}, t_{0}+\frac{\lambda^{2}}{4 N+B}\right)-u^{\varepsilon}\left(x_{0}, t_{0}\right)\right| \leqslant C(\bar{L}) \lambda .
$$

Let $\left(x_{0}, t_{0}+\Delta t\right) \in K$. If $0<\Delta t<\frac{\tau^{2}}{4 N+B}$, we take $\lambda=\Delta t^{1 / 2} \sqrt{4 N+B}<\tau$ to get

$$
\left|u^{\varepsilon}\left(x_{0}, t_{0}+\Delta t\right)-u^{\varepsilon}\left(x_{0}, t_{0}\right)\right| \leqslant C(\bar{L}) \sqrt{4 N+B} \Delta t^{1 / 2} .
$$

If $\Delta t \geqslant \frac{\tau^{2}}{4 N+B}$, we have

$$
\left|u^{\varepsilon}\left(x_{0}, t_{0}+\Delta t\right)-u^{\varepsilon}\left(x_{0}, t_{0}\right)\right| \leqslant 2 \mathcal{A} \leqslant \frac{2 \mathcal{A}}{\tau} \sqrt{4 N+B} \Delta t^{1 / 2} .
$$

The analogous inequality for $v^{\varepsilon}$ is an immediate consequence of (1.6).

REMARK 2.1 Under the hypothesis of the previous propositions, we have that

$$
u^{\varepsilon} \in \operatorname{Lip}_{\text {loc }}\left(1, \frac{1}{2}\right) .
$$

Proposition 2.3 Let $\left(u^{\varepsilon}, v^{\varepsilon}\right)$ be solutions of (1.1), such that $\left\|u^{\varepsilon}\right\|_{\infty} \leqslant \mathcal{A}, v^{\varepsilon} \geqslant 0$, and verify (1.5). Then, for every sequence $\varepsilon_{n} \rightarrow 0$, there exists $\varepsilon_{n^{\prime}} \rightarrow 0$ a subsequence and $u \in \operatorname{Lip}_{\text {loc }}\left(1, \frac{1}{2}\right)$ such that

1. $u^{\varepsilon_{n^{\prime}}} \rightarrow u$ uniformly on compact subsets of $\mathcal{D}$;

2. $\nabla u^{\varepsilon_{n^{\prime}}} \rightarrow \nabla u$ in $L_{\mathrm{loc}}^{2}$;

3. $\frac{\partial u^{\varepsilon} n^{\prime}}{\partial t} \rightarrow \frac{\partial u}{\partial t}$ weakly in $L_{\text {loc }}^{2}$;

4. $\Delta u-\frac{\partial u}{\partial t}=0$ in $\{u>0\}$;

5. for every compact $K \subset \mathcal{D}$, there exists $C_{K}>0$ such that

$$
\left\|\frac{\partial u^{\varepsilon}}{\partial t}\right\|_{L^{2}(K)} \leqslant C_{K}
$$

for every $\varepsilon>0$.

Proof. The proof is similar to Lemma 3.1 of [5].

Let $K \subset \mathcal{D}$ be a compact set and $\tau>0$ such that $\mathcal{N}_{3 \tau}(K) \subset \mathcal{D}$. Let $L=L(K)$ such that

$$
\left|u^{\varepsilon}(x, t)-u^{\varepsilon}(y, s)\right| \leqslant L\left(|x-y|+|t-s|^{1 / 2}\right),
$$

where $(x, t),(y, s) \in \mathcal{N}_{\tau}(K)$.

Then, by Arzela-Ascoli's theorem, there exists $\varepsilon_{n^{\prime}} \rightarrow 0$ and $u \in \operatorname{Lip}\left(1, \frac{1}{2}\right)$ in $\mathcal{N}_{\tau}(K)$ such that $u^{\varepsilon_{n^{\prime}}} \rightarrow u$ uniformly in $\mathcal{N}_{\tau}(K)$. By a standard diagonal argument, (1) follows.

Let us now find uniform bounds for $\frac{\partial u^{\varepsilon}}{\partial t}$ in $L_{\text {loc }}^{2}(\mathcal{D})$. In fact, $u^{\varepsilon}$ verifies

$$
\Delta u^{\varepsilon}-\frac{\partial u^{\varepsilon}}{\partial t}=v^{\varepsilon} f_{\varepsilon}\left(u^{\varepsilon}\right) .
$$


Now, let $\left(x_{0}, t_{0}\right) \in K$ and let us multiply the equation by $u_{t}^{\varepsilon} \psi^{2}$ where $\psi \geqslant 0, \psi=\psi(x) \in$ $C_{c}^{\infty}\left(B_{\tau}\left(x_{0}\right)\right), \psi \equiv 1$ in $B_{\tau / 2}\left(x_{0}\right)$. Then, integrating by parts, we get

$$
\begin{aligned}
\iint_{Q_{\tau}\left(x_{0}, t_{0}\right)}\left(u_{t}^{\varepsilon}\right)^{2} \psi^{2} \mathrm{~d} x \mathrm{~d} t & +\frac{1}{2} \iint_{Q_{\tau}\left(x_{0}, t_{0}\right)}\left(\left|\nabla u^{\varepsilon}\right|^{2}\right)_{t} \psi^{2} \mathrm{~d} x \mathrm{~d} t+2 \iint_{Q_{\tau}\left(x_{0}, t_{0}\right)} \nabla u^{\varepsilon} u_{t}^{\varepsilon} \psi \nabla \psi \mathrm{d} x \mathrm{~d} t \\
& =-\iint_{Q_{\tau}\left(x_{0}, t_{0}\right)} v^{\varepsilon} f_{\varepsilon}\left(u^{\varepsilon}\right) u_{t}^{\varepsilon} \psi^{2} \mathrm{~d} x \mathrm{~d} t .
\end{aligned}
$$

Now we use Young's inequality to obtain

$$
\begin{aligned}
& \frac{1}{2} \iint_{Q_{\tau}\left(x_{0}, t_{0}\right)}\left(u_{t}^{\varepsilon}\right)^{2} \psi^{2} \mathrm{~d} x \mathrm{~d} t+\frac{1}{2} \int_{B_{\tau}\left(x_{0}\right)}\left|\nabla u^{\varepsilon}\left(x_{0}, t_{0}+\tau^{2}\right)\right|^{2} \psi^{2} \mathrm{~d} x \leqslant \\
& \frac{1}{2} \int_{B_{\tau}\left(x_{0}\right)}\left|\nabla u^{\varepsilon}\left(x_{0}, t_{0}-\tau^{2}\right)\right|^{2} \psi^{2} \mathrm{~d} x-\iint_{Q_{\tau}\left(x_{0}, t_{0}\right)} v^{\varepsilon} f_{\varepsilon}\left(u^{\varepsilon}\right) u_{t}^{\varepsilon} \psi^{2} \mathrm{~d} x \mathrm{~d} t \\
& +C \iint_{Q_{\tau}\left(x_{0}, t_{0}\right)}\left|\nabla u^{\varepsilon}\right|^{2}|\nabla \psi|^{2} \mathrm{~d} x \mathrm{~d} t .
\end{aligned}
$$

Then, by Proposition 2.1,

$$
\begin{aligned}
& \int_{B_{\tau / 2}\left(x_{0}\right)} \int_{t_{0}-\tau^{2}}^{t_{0}+\tau^{2}}\left(u_{t}^{\varepsilon}\right)^{2} \mathrm{~d} x \mathrm{~d} t \leqslant \int_{B_{\tau}\left(x_{0}\right)}\left|\nabla u^{\varepsilon}\left(x_{0}, t_{0}-\tau^{2}\right)\right|^{2} \psi^{2} \mathrm{~d} x \\
& +2\left|\iint_{Q_{\tau}\left(x_{0}, t_{0}\right)} v^{\varepsilon} f_{\varepsilon}\left(u^{\varepsilon}\right) u_{t}^{\varepsilon} \psi^{2} \mathrm{~d} x \mathrm{~d} t\right|+C \iint_{Q_{\tau}\left(x_{0}, t_{0}\right)}\left|\nabla u^{\varepsilon}\right|^{2}|\nabla \psi|^{2} \mathrm{~d} x \mathrm{~d} t \\
& \leqslant C(\tau)+2\left|\iint_{Q_{\tau}\left(x_{0}, t_{0}\right)} v^{\varepsilon} f_{\varepsilon}\left(u^{\varepsilon}\right) u_{t}^{\varepsilon} \psi^{2} \mathrm{~d} x \mathrm{~d} t\right| .
\end{aligned}
$$

Hence, it only remains to get bounds on

$$
\iint_{Q_{\tau}} \psi^{2} u_{t}^{\varepsilon} v^{\varepsilon} f_{\varepsilon}\left(u^{\varepsilon}\right) \mathrm{d} x \mathrm{~d} t=I .
$$

Let

$$
\mathcal{G}_{\varepsilon}(u, x, t)=\int_{0}^{u}\left(w^{\varepsilon}(x, t)+s\right) f_{\varepsilon}(s) \mathrm{d} s,
$$

then

so that we get

$$
\frac{\partial}{\partial t}\left(\mathcal{G}_{\varepsilon}\left(u^{\varepsilon}, x, t\right)\right)=\frac{\partial u^{\varepsilon}}{\partial t} v^{\varepsilon} f_{\varepsilon}\left(u^{\varepsilon}\right)+\frac{\partial \mathcal{G}_{\varepsilon}}{\partial t}\left(u^{\varepsilon}, x, t\right)
$$

$$
I=\iint_{Q_{\tau}} \psi^{2} \frac{\partial}{\partial t}\left(\mathcal{G}_{\varepsilon}\left(u^{\varepsilon}, x, t\right)\right) \mathrm{d} x \mathrm{~d} t-\iint_{Q_{\tau}} \psi^{2} \frac{\partial \mathcal{G}_{\varepsilon}}{\partial t}\left(u^{\varepsilon}, x, t\right) \mathrm{d} x \mathrm{~d} t=A-B .
$$

Let us first get bounds on $A$ :

$$
\begin{aligned}
A & =\int_{t_{0}-\tau^{2}}^{t_{0}+\tau^{2}} \int_{B_{\tau}\left(x_{0}\right)} \psi^{2} \frac{\partial}{\partial t}\left(\mathcal{G}_{\varepsilon}\left(u^{\varepsilon}, x, t\right)\right) \mathrm{d} x \mathrm{~d} t=\int_{B_{\tau}\left(x_{0}\right)} \psi^{2}\left[\int_{t_{0}-\tau^{2}}^{t_{0}+\tau^{2}} \frac{\partial}{\partial t}\left(\mathcal{G}_{\varepsilon}\left(u^{\varepsilon}, x, t\right)\right) \mathrm{d} t\right] \mathrm{d} x \\
& =\int_{B_{\tau}\left(x_{0}\right)} \psi^{2}\left[\mathcal{G}_{\varepsilon}\left(u^{\varepsilon}\left(x, t_{0}+\tau^{2}\right), x, t_{0}+\tau^{2}\right)-\mathcal{G}_{\varepsilon}\left(u^{\varepsilon}\left(x, t_{0}-\tau^{2}\right), x, t_{0}-\tau^{2}\right)\right] \mathrm{d} x .
\end{aligned}
$$


Since $u^{\varepsilon} \geqslant-C \varepsilon, f_{\varepsilon}(s)=0$ if $s \geqslant \varepsilon$ and $\left|w^{\varepsilon}\right|=\mathrm{O}(\varepsilon)$, we have

$$
\left|\mathcal{G}_{\varepsilon}\left(u^{\varepsilon}, x, t\right)\right| \leqslant C \varepsilon \int_{-C \varepsilon}^{\varepsilon} f_{\varepsilon}(s) \mathrm{d} s+\int_{-C \varepsilon}^{\varepsilon} s f_{\varepsilon}(s) \mathrm{d} s \leqslant C,
$$

so that

$$
|A| \leqslant C(\tau) .
$$

It only remains to get bounds on $B$. For that purpose, let us first make the following observation:

$$
\left|\frac{\partial \mathcal{G}_{\varepsilon}}{\partial t}\left(u^{\varepsilon}, x, t\right)\right|=\left|\frac{\partial w^{\varepsilon}}{\partial t}(x, t) \int_{0}^{u^{\varepsilon}} f_{\varepsilon}(s) \mathrm{d} s\right| \leqslant \frac{C}{\varepsilon}\left|\frac{\partial w^{\varepsilon}}{\partial t}(x, t)\right| .
$$

By (1.6),

$$
\left|\frac{\partial w^{\varepsilon}}{\partial t}\right| \leqslant C \varepsilon \quad \text { for } \quad(x, t) \in \mathcal{N}_{\tau}(K) .
$$

Therefore, using the fact that $0 \leqslant \psi \leqslant 1$, we get

$$
B \leqslant \frac{C}{\varepsilon} \iint_{Q_{\tau}}\left|\frac{\partial w^{\varepsilon}}{\partial t}(x, t)\right| \mathrm{d} x \mathrm{~d} t \leqslant \frac{C}{\varepsilon}\left|Q_{\tau}\right|\left|\frac{\partial w^{\varepsilon}}{\partial t}\right| \leqslant C(K, \tau) .
$$

Thus,

$$
\int_{B_{\tau / 2}\left(x_{0}\right)} \int_{t_{0}-\tau^{2}}^{t_{0}+\tau^{2}}\left(u_{t}^{\varepsilon}\right)^{2} \mathrm{~d} x \mathrm{~d} t \leqslant C,
$$

with $C$ independent of $\varepsilon$ and $\left(x_{0}, t_{0}\right) \in K$. Now, as $K$ is compact,

$$
\iint_{K}\left(u_{t}^{\varepsilon}\right)^{2} \mathrm{~d} x \mathrm{~d} t \leqslant C,
$$

so that, for a subsequence, $\frac{\partial}{\partial t} u^{\varepsilon_{n^{\prime}}} \rightarrow \frac{\partial}{\partial t} u$ weakly in $L^{2}(K)$ and by a standard diagonal argument, (3) follows.

Let us see that $u$ is a solution of the heat equation in $\{u>0\}$. In fact, from the fact that $u^{\varepsilon} \rightarrow u$ uniformly on compact subsets of $\mathcal{D}$, we deduce that every point $\left(x_{0}, t_{0}\right) \in\{u>0\}$ has a neighbourhood $V$ such that $u^{\varepsilon}(x, t) \geqslant \lambda>0$ for some $\lambda>0$. Therefore, for $\varepsilon<\lambda$, $f_{\varepsilon}\left(u^{\varepsilon}(x, t)\right)=0$ in $V$. Thus, $u^{\varepsilon}$ is caloric in $V$ for every $\varepsilon<\lambda$, and then the same fact holds for $u$.

Let us finally analyse the convergence of the gradients. We already know that $\left\|\nabla u^{\varepsilon}\right\|_{L^{\infty}\left(\mathcal{N}_{\tau}(K)\right)} \leqslant L$. So we can assume that $\nabla u^{\varepsilon} \rightarrow \nabla u$ weakly in $L^{2}\left(\mathcal{N}_{\tau}(K)\right)$. In particular,

$$
\iint_{\mathcal{N}_{\tau}(K)} \phi|\nabla u|^{2} \leqslant \liminf _{\varepsilon \rightarrow 0} \iint_{\mathcal{N}_{\tau}(K)} \phi\left|\nabla u^{\varepsilon}\right|^{2},
$$

for every non-negative $\phi \in L^{\infty}(\mathcal{D})$.

We follow here ideas from [2] and [7] in order to prove that we have strong convergence.

Since $\Delta u-u_{t}=0$ in $\{u>0\}$, if we take $\delta>0$ and multiply this equation by $(u-\delta)^{+} \psi(x)$ with $\psi \in L^{\infty}(\mathcal{D})$ and non-negative, we get, after integration by parts in $Q_{\tau}\left(x_{0}, t_{0}\right)$,

$$
\begin{aligned}
& \iint_{\{u>\delta\}}|\nabla u|^{2} \psi=-\iint_{\{u>\delta\}} u \nabla u \nabla \psi+\delta \iint_{\{u>\delta\}} \nabla u \nabla \psi \\
& -\frac{1}{2} \int_{\{u>\delta\}}(u-\delta)^{2}\left(x, t_{0}+\tau^{2}\right) \psi(x)+\frac{1}{2} \int_{\{u>\delta\}}(u-\delta)^{2}\left(x, t_{0}-\tau^{2}\right) \psi(x) .
\end{aligned}
$$


Now, letting $\delta \rightarrow 0$, we get

$$
\begin{aligned}
\iint_{\{u>0\}}|\nabla u|^{2} \psi= & -\iint_{\{u>0\}} u \nabla u \nabla \psi- \\
& -\frac{1}{2} \int_{\{u>0\}} u^{2}\left(x, t_{0}+\tau^{2}\right) \psi(x)+\frac{1}{2} \int_{\{u>0\}} u^{2}\left(x, t_{0}-\tau^{2}\right) \psi(x) .
\end{aligned}
$$

On the other hand, since $\psi \geqslant 0, f_{\varepsilon} \geqslant 0$ and $u^{\varepsilon} \geqslant-C \varepsilon$, multiplying (1.1) by $\left(u^{\varepsilon}+C \varepsilon\right) \psi$ and integrating by parts, we get

$$
\begin{aligned}
& \iint_{Q_{\tau}\left(x_{0}, t_{0}\right)}\left|\nabla u^{\varepsilon}\right|^{2} \psi \leqslant-\iint_{Q_{\tau}\left(x_{0}, t_{0}\right)} u^{\varepsilon} \nabla u^{\varepsilon} \nabla \psi-C \varepsilon \iint_{Q_{\tau}\left(x_{0}, t_{0}\right)} \nabla u^{\varepsilon} \nabla \psi \\
& -\frac{1}{2} \int_{B_{\tau}\left(x_{0}\right)}\left(u^{\varepsilon}+C \varepsilon\right)^{2}\left(x, t_{0}+\tau^{2}\right) \psi(x)+\frac{1}{2} \int_{B_{\tau}\left(x_{0}\right)}\left(u^{\varepsilon}+C \varepsilon\right)^{2}\left(x, t_{0}-\tau^{2}\right) \psi(x) .
\end{aligned}
$$

Thus,

$$
\limsup _{\varepsilon \rightarrow 0} \iint_{Q_{\tau}\left(x_{0}, t_{0}\right)}\left|\nabla u^{\varepsilon}\right|^{2} \psi \leqslant \iint_{Q_{\tau}\left(x_{0}, t_{0}\right)}|\nabla u|^{2} \psi
$$

so that

$$
\left\|\psi^{1 / 2} \nabla u^{\varepsilon}\right\|_{L^{2}\left(Q_{\tau}\left(x_{0}, t_{0}\right)\right)} \rightarrow\left\|\psi^{1 / 2} \nabla u\right\|_{L^{2}\left(Q_{\tau}\left(x_{0}, t_{0}\right)\right)}
$$

Since, in addition,

$$
\psi^{1 / 2} \nabla u^{\varepsilon} \rightarrow \psi^{1 / 2} \nabla u \quad \text { weakly in } \quad L^{2}\left(Q_{\tau}\left(x_{0}, t_{0}\right)\right)
$$

it follows that

$$
\psi^{1 / 2} \nabla u^{\varepsilon} \rightarrow \psi^{1 / 2} \nabla u \quad \text { in } \quad L^{2}\left(Q_{\tau}\left(x_{0}, t_{0}\right)\right)
$$

Therefore, as $\psi \equiv 1$ in $B_{\tau / 2}\left(x_{0}\right)$,

$$
\nabla u^{\varepsilon} \rightarrow \nabla u \quad \text { in } \quad L^{2}\left(Q_{\tau / 2}\left(x_{0}, t_{0}\right)\right),
$$

and since $K$ is compact, this implies that

$$
\nabla u^{\varepsilon} \rightarrow \nabla u \quad \text { in } \quad L^{2}(K) .
$$

By the same standard diagonal argument used before, the assertion of the theorem follows.

Next we show that the limit function $u$ is a solution of the free- boundary problem in a very weak sense.

Proposition 2.4 Let $\left(u^{\varepsilon_{j}}, v^{\varepsilon_{j}}\right)$ be a family of solutions of (1.1) in a domain $\mathcal{D} \subseteq \mathbb{R}^{N+1}$ such that $u^{\varepsilon_{j}} \rightarrow u$ uniformly on compact subsets of $\mathcal{D}, v^{\varepsilon_{j}} \geqslant 0$, which verifies (1.5). Then, there exists a locally finite measure $\mu$ supported on the free boundary $\mathcal{D} \cap \partial\{u>0\}$ such that $v^{\varepsilon_{j}} f_{\varepsilon_{j}}\left(u^{\varepsilon_{j}}\right) \rightarrow \mu$ weakly in $\mathcal{D}$ and therefore

that is $\forall \phi \in C_{c}^{\infty}(\mathcal{D})$

$$
\Delta u-\frac{\partial u}{\partial t}=\mu \quad \text { in } \quad \mathcal{D}
$$

$$
\iint_{\mathcal{D}}\left(u \phi_{t}-\nabla u \nabla \phi\right) \mathrm{d} x \mathrm{~d} t=\iint_{\mathcal{D}} \phi \mathrm{d} \mu .
$$


Proof. The proof follows as that of Proposition 3.1 of [5].

Now we state an approximation lemma that will be used throughout the rest of the paper.

LEMMA 2.1 Let $\left(u^{\varepsilon_{j}}, v^{\varepsilon_{j}}\right)$ be a family of solutions of (1.1) in a domain $\mathcal{D} \subseteq \mathbb{R}^{N+1}$, such that $u^{\varepsilon_{j}} \rightarrow u$ uniformly on compact subsets of $\mathcal{D}, v^{\varepsilon_{j}} \geqslant 0$, and verifies (1.5). Let $\left(x_{0}, t_{0}\right) \in \mathcal{D} \cap \partial\{u>0\}$ and let $\left(x_{n}, t_{n}\right) \in \mathcal{D} \cap \partial\{u>0\}$ be such that $\left(x_{n}, t_{n}\right) \rightarrow\left(x_{0}, t_{0}\right)$ as $n \rightarrow \infty$. Let $\lambda_{n} \rightarrow 0, u_{\lambda_{n}}(x, t)=$ $\frac{1}{\lambda_{n}} u\left(x_{n}+\lambda_{n} x, t_{n}+\lambda_{n}^{2} t\right)$ and $\left(u^{\varepsilon_{j}}\right) \lambda_{n}(x, t)=\frac{1}{\lambda_{n}} u^{\varepsilon_{j}}\left(x_{n}+\lambda_{n} x, t_{n}+\lambda_{n}^{2} t\right)$. Assume that $u_{\lambda_{n}} \rightarrow U$ as $n \rightarrow \infty$ uniformly on compact sets of $R^{N+1}$. Then, there exists $j(n) \rightarrow \infty$ such that for every $j_{n} \geqslant j(n)$ there holds that $\frac{\varepsilon_{j_{n}}}{\lambda_{n}} \rightarrow 0$ and

1. $\left(u^{\varepsilon_{j_{n}}}\right)_{\lambda_{n}} \rightarrow U$ uniformly on compact sets of $R^{N+1}$;

2. $\nabla\left(u^{\varepsilon_{j n}}\right) \lambda_{n} \rightarrow \nabla U$ in $L_{\text {loc }}^{2}\left(R^{N+1}\right)$;

3. $\frac{\partial}{\partial t}\left(u^{\varepsilon_{j n}}\right)_{\lambda_{n}} \rightarrow \frac{\partial}{\partial t} U$ weakly in $L_{\text {loc }}^{2}\left(R^{N+1}\right)$.

Also, we deduce that

4. $\nabla u_{\lambda_{n}} \rightarrow \nabla U$ in $L_{\text {loc }}^{2}\left(R^{N+1}\right)$;

5. $\frac{\partial}{\partial t} u_{\lambda_{n}} \rightarrow \frac{\partial}{\partial t} U$ weakly in $L_{\text {loc }}^{2}\left(R^{N+1}\right)$.

Proof. The proof is a straightforward adaptation of Lemma 3.2 of [5]. paper.

Now we state some lemmas on special limits of solutions to $P_{\varepsilon}$ that will be used throughout the

LEMMA 2.2 Let $\left(u^{\varepsilon_{j}}, v^{\varepsilon_{j}}\right)$ be a solution to (1.1) in a domain $\mathcal{D} \subset \mathbb{R}^{N+1}$, such that $v^{\varepsilon_{j}} \geqslant 0$, and verifies (1.7) in $\mathcal{D}$ with $w_{0}=$ constant. Let $\left(x_{0}, t_{0}\right) \in \mathcal{D}$ and assume that $u^{\varepsilon_{j}}$ converges to $u=\alpha\left(x-x_{0}\right)_{1}^{+}$uniformly on compact subsets of $\mathcal{D}$, with $\alpha \in \mathbb{R}$ and $\varepsilon_{j} \rightarrow 0$. Then,

$$
0 \leqslant \alpha \leqslant \sqrt{2 M}
$$

where $M=\int_{-w_{0}}^{1}\left(s+w_{0}\right) f(s) \mathrm{d} s$.

Proof. The proof is an adaptation of Proposition 5.2 of [5].

Without loss of generality we may assume that $\left(x_{0}, t_{0}\right)=(0,0)$.

First we see that necessarily $\alpha \geqslant 0$ since $u$ is subcaloric in $\mathcal{D}$ and $u(0,0)=0$. If $\alpha=0$ there is nothing to prove. So let us assume that $\alpha>0$.

Let $\psi \in C_{c}^{\infty}(\mathcal{D})$. Multiplying (1.1) by $u_{x_{1}}^{\varepsilon} \psi$ and integrating by parts we get

$$
\begin{aligned}
\iint_{\mathcal{D}} u_{t}^{\varepsilon_{j}} u_{x_{1}}^{\varepsilon_{j}} \psi= & \frac{1}{2} \iint_{\mathcal{D}}\left|\nabla u^{\varepsilon_{j}}\right|^{2} \psi_{x_{1}}-\iint_{\mathcal{D}} u_{x_{1}}^{\varepsilon_{j}} \nabla u^{\varepsilon_{j}} \nabla \psi \\
& +\iint_{\mathcal{D}} \mathcal{B}_{\varepsilon_{j}}\left(u^{\varepsilon_{j}}, x, t\right) \psi_{x_{1}}+\iint_{\mathcal{D}} w_{x_{1}}^{\varepsilon_{j}}\left(\int_{-w_{0}}^{u^{\varepsilon_{j}}} f_{\varepsilon_{j}}(s) \mathrm{d} s\right) \psi,
\end{aligned}
$$

where $\mathcal{B}_{\varepsilon}(u, x, t)=\int_{-w_{0} \varepsilon}^{u}\left(s+w^{\varepsilon}\right) f_{\varepsilon}(s) \mathrm{d} s$.

In order to pass to the limit in (2.3) we observe that, by Proposition 2.3

$$
\begin{aligned}
\left(u^{\varepsilon_{j}}\right)_{t} & \rightarrow 0 \quad \text { weakly in } \quad L_{\text {loc }}^{2}(\mathcal{D}), \\
\nabla u^{\varepsilon_{j}} \rightarrow \alpha \mathcal{X}_{\left\{x_{1}>0\right\}} e_{1} & \text { in } \quad L_{\text {loc }}^{2}(\mathcal{D}) .
\end{aligned}
$$


On the other hand,

$$
\frac{\nabla w^{\varepsilon_{j}}}{\varepsilon_{j}} \rightarrow 0 \quad \text { uniformly on compact subsets of } \mathcal{D} .
$$

Therefore, in order to pass to the limit in (2.3) we only need to analyse the limit of $\mathcal{B}_{\varepsilon_{j}}\left(u^{\varepsilon_{j}}, x, t\right)$. On one hand, it is easy to see that

$$
\mathcal{B}_{\varepsilon_{j}}\left(u^{\varepsilon_{j}}(x, t), x, t\right) \rightarrow M
$$

for every $(x, t)$ such that $x_{1}>0$. In fact,

$$
\mathcal{B}_{\varepsilon_{j}}\left(u^{\varepsilon_{j}}, x, t\right)=\int_{-w_{0}}^{\frac{u^{\varepsilon_{j}}}{\varepsilon_{j}}}\left(s+\frac{w^{\varepsilon_{j}}}{\varepsilon_{j}}\right) f(s) \mathrm{d} s=\int_{-w_{0}}^{1}\left(s+\frac{w^{\varepsilon_{j}}}{\varepsilon_{j}}\right) f(s) \mathrm{d} s
$$

if $j$ is large enough. Since $\left|\mathcal{B}_{\varepsilon_{j}}\left(u^{\varepsilon_{j}}, x, t\right)\right| \leqslant C$, it holds that (2.4) holds in $L_{\text {loc }}^{1}\left(\left\{x_{1} \geqslant 0\right\}\right)$. On the other hand, there exists $\bar{M}(x, t) \in L^{\infty}(\mathcal{D})$ such that $\mathcal{B}_{\varepsilon_{j}}\left(u^{\varepsilon_{j}}, x, t\right) \rightarrow \bar{M}(x, t)$ weakly in $L_{\text {loc }}^{2}(\mathcal{D})$. Clearly, $\bar{M}(x, t)=M$ in $\left\{x_{1}>0\right\}$. Let us see that $\bar{M}(x, t)=\bar{M}(t)$ in $\left\{x_{1}<0\right\}$. In fact,

$$
\begin{aligned}
\nabla\left(\mathcal{B}_{\varepsilon_{j}}\left(u^{\varepsilon_{j}}(x, t), x, t\right)\right) & =\frac{\partial \mathcal{B}_{\varepsilon_{j}}}{\partial u}\left(u^{\varepsilon_{j}}, x, t\right) \nabla u^{\varepsilon_{j}}+\nabla \mathcal{B}_{\varepsilon_{j}}\left(u^{\varepsilon_{j}}, x, t\right) \\
& =\left(u^{\varepsilon_{j}}+w^{\varepsilon_{j}}\right) f_{\varepsilon_{j}}\left(u^{\varepsilon_{j}}\right) \nabla u^{\varepsilon_{j}}+\nabla w^{\varepsilon_{j}} \int_{-w_{0} \varepsilon_{j}}^{u^{\varepsilon_{j}}} f_{\varepsilon_{j}}(s) \mathrm{d} s \\
& =v^{\varepsilon_{j}} f_{\varepsilon_{j}}\left(u^{\varepsilon_{j}}\right) \nabla u^{\varepsilon_{j}}+\frac{\nabla w^{\varepsilon_{j}}}{\varepsilon_{j}} \int_{-w_{0}}^{\frac{u^{\varepsilon_{j}}}{\varepsilon_{j}}} f(s) \mathrm{d} s .
\end{aligned}
$$

Since $v^{\varepsilon_{j}} f_{\varepsilon_{j}}\left(u^{\varepsilon_{j}}\right) \rightarrow 0$ in $L_{\text {loc }}^{1}\left(\left\{x_{1}<0\right\}\right), \nabla u^{\varepsilon_{j}}$ is uniformly bounded in $L^{\infty}\left(\mathcal{D}^{\prime}\right)$ if $\mathcal{D}^{\prime} \subset \subset \mathcal{D}$ and $\frac{\nabla w^{\varepsilon_{j}}}{\varepsilon_{j}} \rightarrow 0$ uniformly on compact subsets of $\mathcal{D}$, it holds that

$$
\nabla\left(\mathcal{B}_{\varepsilon_{j}}\left(u^{\varepsilon_{j}}(x, t), x, t\right)\right) \rightarrow 0 \quad \text { in } \quad L_{\mathrm{loc}}^{1}\left(\left\{x_{1}<0\right\}\right) .
$$

So that, passing to the limit in (2.3) we get

$$
\frac{\alpha^{2}}{2} \iint_{\left\{x_{1}>0\right\}} \psi_{x_{1}}=M \iint_{\left\{x_{1}>0\right\}} \psi_{x_{1}}+\int_{\left\{x_{1}<0\right\}} \bar{M}(t) \psi_{x_{1}} .
$$

Thus, integrating in the variable $x_{1}$ we get

$$
\int_{\left\{x_{1}=0\right\}}\left(\frac{\alpha^{2}}{2}-M+\bar{M}(t)\right) \psi=0 .
$$

Since $\psi$ is arbitrary, we conclude that

$$
\frac{\alpha^{2}}{2}-M+\bar{M}(t)=0
$$


Finally, we notice that $\bar{M}(t) \geqslant 0$. In fact,

$$
\begin{gathered}
\mathcal{B}_{\varepsilon_{j}}\left(u^{\varepsilon_{j}}, x, t\right)=\int_{-\frac{w^{\varepsilon_{j}}}{\varepsilon_{j}}}^{\frac{u^{\varepsilon_{j}}}{\varepsilon_{j}}}\left(s+\frac{w^{\varepsilon_{j}}}{\varepsilon_{j}}\right) f(s) \mathrm{d} s+\int_{-w_{0}}^{-\frac{w^{\varepsilon_{j}}}{\varepsilon_{j}}}\left(s+\frac{w^{\varepsilon_{j}}}{\varepsilon_{j}}\right) f(s) \mathrm{d} s \\
\geqslant \int_{-w_{0}}^{-\frac{w^{\varepsilon_{j}}}{\varepsilon_{j}}}\left(s+\frac{w^{\varepsilon_{j}}}{\varepsilon_{j}}\right) f(s) \mathrm{d} s \rightarrow 0
\end{gathered}
$$

since $\frac{w^{\varepsilon_{j}}}{\varepsilon_{j}} \rightarrow w_{0}$ uniformly on compact subsets of $\mathcal{D}$.

Thus,

$$
\alpha=\sqrt{2(M-\bar{M}(t))} \leqslant \sqrt{2 M}
$$

and the proof is complete.

LEMmA 2.3 Let $\left(u^{\varepsilon_{j}}, v^{\varepsilon_{j}}\right)$ be a solution to (1.1) in a domain $\mathcal{D} \subset \mathbb{R}^{N+1}$, such that $v^{\varepsilon_{j}} \geqslant 0$, and verifies (1.7) with $w_{0}=$ constant in $\mathcal{D}$. Let $\left(x_{0}, t_{0}\right) \in \mathcal{D}$ and assume that $u^{\varepsilon_{j}}$ converges to $u=\alpha\left(x-x_{0}\right)_{1}^{+}+\bar{\alpha}\left(x-x_{0}\right)_{1}^{-}$uniformly on compact subsets of $\mathcal{D}$, with $\alpha, \bar{\alpha}>0$ and $\varepsilon_{j} \rightarrow 0$. Then,

$$
\bar{\alpha}=\alpha \leqslant \sqrt{2 M}
$$

where $M=\int_{-w_{0}}^{1}\left(s+w_{0}\right) f(s) \mathrm{d} s$.

Proof. We argue in a similar way as in Proposition 5.3 of [5].

We will denote $Q_{r}=Q_{r}(0,0)$. Without loss of generality we will assume that $\left(x_{0}, t_{0}\right)=(0,0)$ and that $Q_{2} \subset \subset \mathcal{D}$.

As before, $u^{\varepsilon}$ satisfies

$$
\begin{aligned}
\iint_{\mathcal{D}} u_{t}^{\varepsilon} u_{x_{1}}^{\varepsilon} \psi= & \frac{1}{2} \iint_{\mathcal{D}}\left|\nabla u^{\varepsilon}\right|^{2} \psi_{x_{1}}-\iint_{\mathcal{D}} u_{x_{1}}^{\varepsilon} \nabla u^{\varepsilon} \nabla \psi \\
& +\iint_{\mathcal{D}} \mathcal{B}_{\varepsilon}\left(u^{\varepsilon}, x, t\right) \psi_{x_{1}}+\iint_{\mathcal{D}} w_{x_{1}}^{\varepsilon}\left(\int_{0}^{u^{\varepsilon}} f_{\varepsilon}(s) \mathrm{d} s\right) \psi
\end{aligned}
$$

We want to pass to the limit. By Proposition 2.3 and the fact that $u^{\varepsilon_{j}}$ converges to $\alpha x_{1}^{+}+\bar{\alpha} x_{1}^{-}$ we have that

$$
\begin{gathered}
u_{t}^{\varepsilon_{j}} \rightarrow 0 \quad \text { weakly in } L_{\mathrm{loc}}^{2}(\mathcal{D}), \\
\nabla u^{\varepsilon_{j}} \rightarrow \alpha \mathcal{X}_{\left\{x_{1}>0\right\}} e_{1}-\bar{\alpha} \mathcal{X}_{\left\{x_{1}<0\right\}} e_{1} \quad \text { in } L_{\mathrm{loc}}^{2}(\mathcal{D}) .
\end{gathered}
$$

Clearly, as $\alpha, \bar{\alpha}>0, \mathcal{B}\left(u^{\varepsilon_{j}}, x, t\right) \rightarrow M$ in $L_{\text {loc }}^{1}(\mathcal{D})$.

So, passing to the limit in the latter equation for the subsequence $\varepsilon_{j}$, we get

$$
-\frac{\alpha^{2}}{2} \iint_{\left\{x_{1}>0\right\}} \psi_{x_{1}}-\frac{\bar{\alpha}^{2}}{2} \iint_{\left\{x_{1}<0\right\}} \psi_{x_{1}}+M \iint \psi_{x_{1}}=0 .
$$

Integrating in the $x_{1}$ variable, we conclude that

$$
\alpha=\bar{\alpha} .
$$


Next, we will assume that $\alpha>\sqrt{2 M}$ and arrive at a contradiction.

First, let us consider $z^{\varepsilon_{j}}$, defined in $Q_{2}$, the solution to

$$
\Delta z^{\varepsilon_{j}}-z_{t}^{\varepsilon_{j}}=\left(\beta_{\varepsilon_{j}}\left(z^{\varepsilon_{j}}\right)+W_{\varepsilon_{j}} f_{\varepsilon_{j}}\left(z^{\varepsilon_{j}}\right)\right) \rho_{\varepsilon_{j}}\left(z^{\varepsilon_{j}} / \varepsilon_{j}\right) \quad \text { in } \quad Q_{2}
$$

with boundary conditions

$$
z^{\varepsilon_{j}}=u-b^{\varepsilon_{j}} \quad \text { on } \quad \partial_{p} Q_{2}
$$

where $\beta_{\varepsilon}(s)=s f_{\varepsilon}(s), W_{\varepsilon}=\sup _{Q_{2}} w^{\varepsilon}, b^{\varepsilon_{j}}=\sup _{Q_{2}}\left|u^{\varepsilon_{j}}-u\right|$ and $\rho_{\varepsilon_{j}}$ is a smooth cutoff function with support in $\left[-\left(w_{0}+2 C_{\varepsilon_{j}}\right), 2\right]$ and $\rho_{\varepsilon_{j}} \equiv 1$ in $\left[-\left(w_{0}+C_{\varepsilon_{j}}\right)\right.$, 1]. (Here $C_{\varepsilon_{j}} \rightarrow 0^{+}$is such that $\left|w^{\varepsilon_{j}} / \varepsilon_{j}-w_{0}\right| \leqslant C_{\varepsilon_{j}}$ in $Q_{2}$ so that $u^{\varepsilon_{j}} / \varepsilon_{j} \geqslant-\left(w_{0}+C_{\varepsilon_{j}}\right)$ in $Q_{2}$.)

We observe that $z^{\varepsilon_{j}}\left(x_{1}, x^{\prime}, t\right)=z^{\varepsilon_{j}}\left(-x_{1}, x^{\prime}, t\right)$ in $Q_{2}$.

It is easy to see that the proofs of Propositions 2.1 and 2.2 can be adapted to $z^{\varepsilon_{j}}$ so that, for a subsequence, that we still call $\varepsilon_{j}$, it holds that $z^{\varepsilon_{j}} \rightarrow z$ uniformly on compact sets of $Q_{2}$. We will show that $z=u$.

First,

$$
\begin{aligned}
\Delta u^{\varepsilon_{j}}-u_{t}^{\varepsilon_{j}} & =v^{\varepsilon_{j}} f_{\varepsilon_{j}}\left(u^{\varepsilon_{j}}\right)=\left(u^{\varepsilon_{j}}+w^{\varepsilon_{j}}\right) f_{\varepsilon_{j}}\left(u^{\varepsilon_{j}}\right) \leqslant \beta_{\varepsilon_{j}}\left(u^{\varepsilon_{j}}\right)+W_{\varepsilon_{j}} f_{\varepsilon_{j}}\left(u^{\varepsilon_{j}}\right) \\
& =\left(\beta_{\varepsilon_{j}}\left(u^{\varepsilon_{j}}\right)+W_{\varepsilon_{j}} f_{\varepsilon_{j}}\left(u^{\varepsilon_{j}}\right)\right) \rho_{\varepsilon_{j}}\left(u^{\varepsilon_{j}} / \varepsilon_{j}\right) \text { in } Q_{2} .
\end{aligned}
$$

From the fact that $z^{\varepsilon_{j}} \leqslant u^{\varepsilon_{j}}$ on $\partial_{p} Q_{2}$, we deduce that $z^{\varepsilon_{j}} \leqslant u^{\varepsilon_{j}}$ in $Q_{2}$ and therefore $z \leqslant u$. In order to see that $u \leqslant z$, we consider $a^{\varepsilon_{j}} \in C^{2}(\mathbb{R})$ such that

$$
\begin{aligned}
& a_{s s}^{\varepsilon_{j}}=\left(\beta\left(a^{\varepsilon_{j}}\right)+\frac{W_{\varepsilon_{j}}}{\varepsilon_{j}} f\left(a^{\varepsilon_{j}}\right)\right) \rho_{\varepsilon_{j}}\left(a^{\varepsilon_{j}}\right), \quad s \in \mathbb{R} \\
& a^{\varepsilon_{j}}(0)=1, \quad a_{s}^{\varepsilon_{j}}(0)=\alpha .
\end{aligned}
$$

Integrating the equation we get, for every $s \in \mathbb{R}$,

$$
0<\gamma-\kappa_{\varepsilon_{j}} \leqslant a_{s}^{\varepsilon_{j}}(s) \leqslant \alpha
$$

where $\frac{1}{2} \gamma^{2} \equiv \frac{1}{2} \alpha^{2}-M>0$ and $\kappa_{\varepsilon_{j}} \rightarrow 0$ when $j \rightarrow \infty$.

It follows that there exists $\bar{s}_{\varepsilon_{j}}<0$ such that

$$
a^{\varepsilon_{j}}(s)= \begin{cases}1+\alpha s & s \geqslant 0 \\ \left(\gamma-\kappa_{\varepsilon_{j}}\right)\left(s-\bar{s}_{\varepsilon_{j}}\right) & s \leqslant \bar{s}_{\varepsilon_{j}}\end{cases}
$$

and it is easy to see that $\bar{s}_{\varepsilon_{j}}$ are uniformly bounded below and, moreover, there exists $\bar{s}<0$ such that $\bar{s}_{\varepsilon_{j}} \rightarrow \bar{s}$.

Now let

$$
\tilde{a}^{\varepsilon_{j}}(x)=\varepsilon_{j} a^{\varepsilon_{j}}\left(\frac{x_{1}}{\varepsilon_{j}}-\frac{b^{\varepsilon_{j}}}{\left(\gamma-\kappa_{\varepsilon_{j}}\right) \varepsilon_{j}}+\bar{s}_{\varepsilon_{j}}\right) .
$$

Using that $\tilde{a}^{\varepsilon_{j}}\left(0, x^{\prime}, t\right)=-b^{\varepsilon_{j}}$ and the bounds on $a_{s}^{\varepsilon_{j}}$, we deduce that

$$
\tilde{a}^{\varepsilon_{j}} \leqslant u-b^{\varepsilon_{j}} \quad \text { in } \quad Q_{2} .
$$

Now, since $\tilde{a}^{\varepsilon_{j}} \leqslant z^{\varepsilon_{j}}$ on $\partial_{p} Q_{2}$, and $\tilde{a}^{\varepsilon_{j}}$ is a one-dimensional stationary solution to (2.6), we have that $\tilde{a}^{\varepsilon_{j}} \leqslant z^{\varepsilon_{j}}$ in $Q_{2}$. Since $\tilde{a}^{\varepsilon_{j}} \rightarrow u$ uniformly on compact subsets of $\left\{x_{1}>0\right\}$, we deduce that $u \leqslant z$ in $Q_{2} \cap\left\{x_{1}>0\right\}$. 
Finally, we notice that $z^{\varepsilon_{j}}\left(x_{1}, x^{\prime}, t\right)=z^{\varepsilon_{j}}\left(-x_{1}, x^{\prime}, t\right)$, so we conclude that $u \leqslant z$ in $Q_{2}$.

Now, the proof of the lemma follows as in [5] where it is shown, for the case in which $w^{\varepsilon} \equiv 0$, that if $z^{\varepsilon_{j}} \rightarrow \alpha x_{1}^{+}+\alpha x_{1}^{-}$uniformly on compact subsets of $Q_{2}$, where $z^{\varepsilon_{j}}$ are solutions to (2.6) which are symmetric in the $x_{1}$ variable, and $\alpha>0$, it holds that $\alpha \leqslant \sqrt{2 M}$. So that we arrive at a contradiction since we have assumed that $\alpha>\sqrt{2 M}$. Here we use again that $\frac{w^{\varepsilon_{j}}}{\varepsilon_{j}} \rightarrow w_{0}$ and $\lim \inf \mathcal{B}_{\varepsilon_{j}}\left(u^{\varepsilon_{j}}, x, t\right) \geqslant 0$. The proof is complete.

Lemma 2.4 Let $\left(u^{\varepsilon_{j}}, v^{\varepsilon_{j}}\right)$ be a solution to (1.1) in a domain $\mathcal{D}_{j}$ such that $v^{\varepsilon_{j}} \geqslant 0$, and satisfies (1.7) in $\mathcal{D}_{j}$ with $w_{0}=$ constant. Here $\mathcal{D}_{j}$ is such that $\mathcal{D}_{j} \subset \mathcal{D}_{j+1}$ and $\cup_{j} \mathcal{D}_{j}=\mathbb{R}^{N+1}$. Let us assume that $u^{\varepsilon_{j}} \rightarrow U$ uniformly on compact subsets of $\mathbb{R}^{N+1}$ as $j \rightarrow \infty$ and $\varepsilon_{j} \rightarrow 0$, with $U \geqslant 0, U \in \operatorname{Lip}\left(1, \frac{1}{2}\right)$ and $\partial\{U>0\} \neq \emptyset$. Then,

$$
|\nabla U| \leqslant \sqrt{2 M} \quad \text { in } \quad \mathbb{R}^{N+1}
$$

with $M=\int_{-w_{0}}^{1}\left(s+w_{0}\right) f(s) \mathrm{d} s$.

Proof. The proof is similar to that of Theorem 6.2 in [5]. Here we use Lemmas 2.2 and 2.3 instead of Propositions 5.2 and 5.3 in [5].

\section{The free-boundary condition}

In this section, we find the free-boundary condition for the limit problem and we show that the limit function $u$ is a solution to the free-boundary problem (1.4) in a pointwise sense, under the assumption that the free boundary admits an inward spatial normal in a parabolic measure theoretic sense (Definition 3.1).

Throughout this section we will assume that (1.7) holds and that for every $K \subset\{u \equiv 0\}^{\circ}$ compact there exists $0<\eta<1$ and $\varepsilon_{0}>0$ such that, for $\varepsilon<\varepsilon_{0}$,

$$
\frac{u^{\varepsilon}}{\varepsilon} \leqslant \eta \quad \text { in } \quad K
$$

This assumption is a natural one in applications; roughly speaking it means that the mixture temperature reaches the flame temperature only if some combustion is taking place.

As a consequence, it holds that

$$
w_{0}=\lim _{\varepsilon \rightarrow 0} \frac{v^{\varepsilon}-u^{\varepsilon}}{\varepsilon} \geqslant-\limsup _{\varepsilon \rightarrow 0} \frac{u^{\varepsilon}}{\varepsilon} \geqslant-\eta>-1 \quad \text { in } \quad K .
$$

So that, for the sake of simplicity, we will assume from now on that $w_{0}>-1$ in $\mathcal{D}$.

We start this section with a lemma that is the essential ingredient in the subsequent proofs.

LEMMA 3.1 Let $\left(u^{\varepsilon_{k}}, v^{\varepsilon_{k}}\right)$ be a solution to (1.1) in a domain $\mathcal{D} \subset \mathbb{R}^{N+1}$ such that $v^{\varepsilon_{k}} \geqslant 0$ and (1.7) and (3.1) are satisfied with $w_{0}>-1$. Let $u=\lim u^{\varepsilon_{k}}$, with $\varepsilon_{k} \rightarrow 0$, and $\mathcal{B}_{\varepsilon_{k}}(u, x, t)=\int_{-w_{0} \varepsilon_{k}}^{u}(s+$ $\left.w^{\varepsilon_{k}}\right) f_{\varepsilon_{k}}(s) \mathrm{d} s$. Then,

$$
\mathcal{B}_{\varepsilon_{k}}\left(u^{\varepsilon_{k}}, x, t\right) \rightarrow M(x, t) \mathcal{X}_{\{u>0\}}, \quad \text { in } \quad L_{\mathrm{loc}}^{1}(\mathcal{D})
$$

where $M(x, t)=\int_{-w_{0}(x, t)}^{1}\left(s+w_{0}(x, t)\right) f(s) \mathrm{d} s$. 
Proof. First, let us observe that

$$
\begin{aligned}
\int_{-w_{0} \varepsilon}^{\varepsilon}\left(w^{\varepsilon}+s\right) f_{\varepsilon}(s) \mathrm{d} s & =\int_{-w_{0} \varepsilon}^{\varepsilon}\left(w^{\varepsilon}+s\right) \frac{1}{\varepsilon^{2}} f\left(\frac{s}{\varepsilon}\right) \mathrm{d} s \\
& =\int_{-w_{0}}^{1}\left(\frac{w^{\varepsilon}}{\varepsilon}+s\right) f(s) \mathrm{d} s .
\end{aligned}
$$

Therefore,

$$
\lim _{\varepsilon_{k} \rightarrow 0} \int_{-w_{0} \varepsilon_{k}}^{\varepsilon_{k}}\left(w^{\varepsilon_{k}}+s\right) f_{\varepsilon_{k}}(s) \mathrm{d} s=M(x, t) .
$$

Let us now see that $\mathcal{B}_{\varepsilon_{k}}\left(u^{\varepsilon_{k}}, x, t\right) \rightarrow M(x, t)$ uniformly on compact subsets of $\{u>0\}$.

Let $K \subset \subset\{u>0\}$, then there exists $\lambda>0$ and $\varepsilon_{0}$ such that $u^{\varepsilon_{k}}(x, t)>\lambda \forall \varepsilon_{k}<\varepsilon_{0},(x, t) \in$ $K$. Thus, we have

$$
\begin{aligned}
\lim _{k \rightarrow \infty} \mathcal{B}_{\varepsilon_{k}}\left(u^{\varepsilon_{k}}(x, t), x, t\right) & =\lim _{k \rightarrow \infty} \int_{-w_{0} \varepsilon_{k}}^{u^{\varepsilon_{k}}(x, t)}\left(w^{\varepsilon}+s\right) f_{\varepsilon_{k}}(s) \mathrm{d} s \\
& =\lim _{k \rightarrow \infty} \int_{-w_{0} \varepsilon_{k}}^{\varepsilon_{k}}\left(w^{\varepsilon}+s\right) f_{\varepsilon_{k}}(s) \mathrm{d} s=M(x, t) .
\end{aligned}
$$

Since $\left|\mathcal{B}_{\varepsilon_{k}}\left(u^{\varepsilon_{k}}, x, t\right)\right| \leqslant C$ on every compact subset of $\mathcal{D}$, it holds, for a subsequence that we still call $\varepsilon_{k}$, that

$$
\mathcal{B}_{\varepsilon_{k}}\left(u^{\varepsilon_{k}}, x, t\right) \rightarrow \bar{M}(x, t) \quad \text { weakly in } \quad L_{\text {loc }}^{2}(\mathcal{D}) .
$$

Clearly, $\bar{M}(x, t)=M(x, t)$ in $\{u>0\}$. Let us see that $\bar{M}(x, t)=0$ in $\{u \equiv 0\}^{\circ}$. In fact, let $K$ be a compact subset of $\{u \equiv 0\}^{\circ}$. For every $\varepsilon_{1}, \varepsilon_{2}>0$, it holds that, for $k$ large enough,

$$
\begin{aligned}
& \left|\left\{(x, t) \in K / \varepsilon_{1}<\mathcal{B}_{\varepsilon_{k}}\left(u^{\varepsilon_{k}}, x, t\right)<M(x, t)-\varepsilon_{2}\right\}\right| \\
& \quad \leqslant\left|\left\{(x, t) \in K / \frac{u^{\varepsilon_{k}}}{\varepsilon_{k}}(x, t)>-w_{0}(x, t), \frac{\varepsilon_{1}}{2}<\int_{-w_{0}}^{\frac{u^{\varepsilon_{k}}}{\varepsilon_{k}}}\left(s+w_{0}\right) f(s) \mathrm{d} s<M-\frac{\varepsilon_{2}}{2}\right\}\right| \\
& \quad \leqslant\left|\left\{(x, t) \in K /-w_{0}(x, t)+\mu \leqslant \frac{u^{\varepsilon_{k}}}{\varepsilon_{k}} \leqslant 1-\mu\right\}\right| \\
& \quad \leqslant\left|\left\{(x, t) \in K / \frac{v^{\varepsilon_{k}}}{\varepsilon_{k}} \geqslant \frac{\mu}{2}, \frac{u^{\varepsilon_{k}}}{\varepsilon_{k}} \leqslant 1-\mu\right\}\right| \\
& \quad \leqslant\left|\left\{(x, t) \in K / v^{\varepsilon_{k}} f_{\varepsilon_{k}}\left(u^{\varepsilon_{k}}\right) \geqslant \frac{C_{\mu}}{2 \varepsilon_{k}}\right\}\right| .
\end{aligned}
$$

Since $v^{\varepsilon_{k}} f_{\varepsilon_{k}}\left(u^{\varepsilon_{k}}\right) \rightarrow 0$ as measures in $K$ and $v^{\varepsilon_{k}} \geqslant 0, f_{\varepsilon_{k}} \geqslant 0$, it holds that

$$
v^{\varepsilon_{k}} f_{\varepsilon_{k}}\left(u^{\varepsilon_{k}}\right) \rightarrow 0 \quad \text { in } \quad L^{1}(K) .
$$

Therefore,

$$
\left|\left\{(x, t) \in K / \varepsilon_{1}<\mathcal{B}_{\varepsilon_{k}}\left(u^{\varepsilon_{k}}, x, t\right)<M(x, t)-\varepsilon_{2}\right\}\right| \rightarrow 0 .
$$


On the other hand, let $1>\eta>\sup _{K}\left(-w_{0}\right)$ be the constant in (3.1) in $K$. It holds that

$$
\begin{gathered}
\mathcal{B}_{\varepsilon_{k}}\left(u^{\varepsilon_{k}}, x, t\right)=\int_{-\frac{w^{\varepsilon_{k}}}{\varepsilon_{k}}}^{\frac{u^{\varepsilon_{k}}}{\varepsilon_{k}}}\left(s+\frac{w^{\varepsilon_{k}}}{\varepsilon_{k}}\right) f(s) \mathrm{d} s+\int_{-w_{0}}^{-\frac{w^{\varepsilon_{k}}}{\varepsilon_{k}}}\left(s+\frac{w^{\varepsilon_{k}}}{\varepsilon_{k}}\right) f(s) \mathrm{d} s \\
\leqslant \int_{-\frac{w^{\varepsilon_{k}}}{\varepsilon_{k}}}^{\eta}\left(s+\frac{w^{\varepsilon_{k}}}{\varepsilon_{k}}\right) f(s) \mathrm{d} s+\int_{-w_{0}}^{-\frac{w^{\varepsilon_{k}}}{\varepsilon_{k}}}\left(s+\frac{w^{\varepsilon_{k}}}{\varepsilon_{k}}\right) f(s) \mathrm{d} s \\
=\int_{-w_{0}}^{\eta}\left(s+\frac{w^{\varepsilon_{k}}}{\varepsilon_{k}}\right) f(s) \mathrm{d} s \rightarrow \int_{-w_{0}}^{\eta}\left(s+w_{0}\right) f(s) \mathrm{d} s<M(x, t)
\end{gathered}
$$

since $-\frac{w^{\varepsilon_{k}}}{\varepsilon_{k}} \leqslant \frac{u^{\varepsilon_{k}}}{\varepsilon_{k}} \leqslant \eta$ in $K$. Therefore,

$$
\lim \sup \mathcal{B}_{\varepsilon_{k}}\left(u^{\varepsilon_{k}}, x, t\right) \leqslant \int_{-w_{0}}^{\eta}\left(s+w_{0}\right) f(s) \mathrm{d} s<M(x, t) .
$$

So that, for $\varepsilon_{2}>0$ small, we get

$$
\left|\left\{(x, t) \in K / \varepsilon_{1}<\mathcal{B}_{\varepsilon_{k}}\left(u^{\varepsilon_{k}}, x, t\right)\right\}\right|=\left|\left\{(x, t) \in K / \varepsilon_{1}<\mathcal{B}_{\varepsilon_{k}}\left(u^{\varepsilon_{k}}, x, t\right)<M-\varepsilon_{2}\right\}\right| \rightarrow 0 .
$$

Let us now see that $\bar{M}(x, t)=0$ in $K$. As in Lemma 2.2 we see that $\bar{M}(x, t) \geqslant 0$. Now assume that for some $\varepsilon_{1}>0$ we have $\left|\left\{\bar{M}(x, t)>\varepsilon_{1}\right\}\right|>0$. Then, there exists $m$ such that $\left|\left\{\bar{M}(x, t)>\varepsilon_{1}+\frac{1}{m}\right\}\right|:=\left|A_{m}\right|>0$.

Now,

$$
\int_{A_{m}} \mathcal{B}_{\varepsilon_{k}}\left(u^{\varepsilon_{k}}, x, t\right) \rightarrow \int_{A_{m}} \bar{M}(x, t)>\left(\varepsilon_{1}+\frac{1}{m}\right)\left|A_{m}\right|
$$

but,

$$
\begin{aligned}
\int_{A_{m}} \mathcal{B}_{\varepsilon_{k}}\left(u^{\varepsilon_{k}}, x, t\right) & =\int_{A_{m} \cap\left\{\mathcal { B } _ { \varepsilon _ { k } } \left(u^{\left.\left.\varepsilon_{k}, x, t\right)>\varepsilon_{1}\right\}}\right.\right.} \mathcal{B}_{\varepsilon_{k}}\left(u^{\varepsilon_{k}}, x, t\right) \\
& +\int_{A_{m} \cap\left\{\mathcal{B}_{\varepsilon_{k}}\left(u^{\varepsilon}, x, t\right) \leqslant \varepsilon_{1}\right\}} \mathcal{B}_{\varepsilon_{k}}\left(u^{\varepsilon_{k}}, x, t\right) .
\end{aligned}
$$

Since the first term in the right-hand side goes to zero and the second is bounded by $\varepsilon_{1}\left|A_{m}\right|$, we get a contradiction.

The proof is complete.

Let us give the definition of a regular point.

DEFINITION 3.1 We say that $v$ is the interior unit spatial normal to the free boundary $\partial\{u>0\}$ at a point $\left(x_{0}, t_{0}\right) \in \partial\{u>0\}$ in the parabolic measure theoretic sense, if $v \in \mathbb{R}^{N},|v|=1$ and

$$
\lim _{r \rightarrow 0} \frac{1}{r^{N+2}} \iint_{Q_{r}\left(x_{0}, t_{0}\right)}\left|\mathcal{X}_{\{u>0\}}-\mathcal{X}_{\left\{(x, t) /\left\langle x-x_{0}, \nu\right\rangle>0\right\}}\right| \mathrm{d} x \mathrm{~d} t=0 .
$$

DEFINITION 3.2 We say that $\left(x_{0}, t_{0}\right)$ is a regular point of $\partial\{u>0\}$ if there exists an interior unit spatial normal to $\partial\{u>0\}$ at $\left(x_{0}, t_{0}\right)$ in the parabolic measure theoretic sense. 
We can now prove the main result of this section.

THEOREM 3.1 Let $\left(u^{\varepsilon_{j}}, v^{\varepsilon_{j}}\right)$ be a family of uniformly bounded solutions of (1.1) in a domain $\mathcal{D} \subset \mathbb{R}^{N+1}$, such that $u^{\varepsilon_{j}} \rightarrow u$ uniformly on a compact subset of $\mathcal{D}, v^{\varepsilon_{j}} \geqslant 0$, and verifies (1.7) and (3.1), with $w_{0}>-1$. If $\left(x_{0}, t_{0}\right)$ is a regular point of $\mathcal{D} \cap \partial\{u>0\}$, then $u$ has the asymptotic development

$$
u(x, t)=\alpha\left\langle x-x_{0}, v\right\rangle^{+}+\mathrm{o}\left(\left|x-x_{0}\right|+\left|t-t_{0}\right|^{1 / 2}\right)
$$

with $\alpha=\sqrt{2 M\left(x_{0}, t_{0}\right)}$, where $M=\int_{-w_{0}}^{1}\left(s+w_{0}\right) f(s) \mathrm{d} s$. Here $v$ is the interior unit spatial normal to the free boundary at $\left(x_{0}, t_{0}\right)$ in the parabolic measure theoretic sense.

Proof. We assume, without loss of generality, that $\left(x_{0}, t_{0}\right)=(0,0)$ and $v=e_{1}=(1,0, \ldots, 0)$.

Let $\psi \in C_{c}^{\infty}(\mathcal{D})$. We proceed as in Lemma 2.2. Let us multiply the equation for $u^{\varepsilon}$ by $u_{x_{1}}^{\varepsilon} \psi$ and integrate by parts. We have

$$
\begin{aligned}
\iint_{\mathcal{D}} u_{t}^{\varepsilon} u_{x_{1}}^{\varepsilon} \psi= & \frac{1}{2} \iint_{\mathcal{D}}\left|\nabla u^{\varepsilon}\right|^{2} \psi_{x_{1}}-\iint_{\mathcal{D}} u_{x_{1}}^{\varepsilon} \nabla u^{\varepsilon} \nabla \psi \\
& +\iint_{\mathcal{D}} \mathcal{B}_{\varepsilon}\left(u^{\varepsilon}, x, t\right) \psi_{x_{1}}+\iint_{\mathcal{D}}\left(\frac{w^{\varepsilon}}{\varepsilon}-w_{0}\right) f\left(-w_{0}\right)\left(w_{0}\right)_{x_{1}} \psi \\
& +\iint_{\mathcal{D}} \frac{w_{x_{1}}^{\varepsilon}}{\varepsilon}\left(\int_{-w_{0}}^{\frac{u^{\varepsilon}}{\varepsilon}} f(s) \mathrm{d} s\right) \psi
\end{aligned}
$$

Since

$$
\mathcal{B}_{\varepsilon_{j}}\left(u^{\varepsilon_{j}}, x, t\right)=\int_{-w_{0}}^{\frac{u^{\varepsilon_{j}}}{\varepsilon_{j}}}\left(s+w_{0}\right) f(s) \mathrm{d} s+\int_{-w_{0}}^{\frac{u^{\varepsilon_{j}}}{\varepsilon_{j}}}\left(\frac{w^{\varepsilon_{j}}}{\varepsilon_{j}}-w_{0}\right) f(s) \mathrm{d} s,
$$

and $\mathcal{B}_{\varepsilon_{j}}\left(u^{\varepsilon_{j}}, x, t\right) \rightarrow 0$ weakly in $L^{1}(K)$ for every $K \subset\{u \equiv 0\}^{\circ}$ compact, it holds that

$$
F_{\varepsilon_{j}}(x, t):=\int_{-w_{0}}^{\frac{u^{\varepsilon_{j}}}{\varepsilon_{j}}}\left(s+w_{0}\right) f(s) \mathrm{d} s \rightarrow 0 \quad \text { weakly in } \quad L^{1}(K) .
$$

Since $F_{\varepsilon_{j}}$ is non-negative, it holds that

$$
F_{\varepsilon_{j}} \rightarrow 0 \quad \text { in } \quad L^{1}(K)
$$

So that, for a subsequence that we still call $\varepsilon_{j}$, it holds that

$$
F_{\varepsilon_{j}} \rightarrow 0 \quad \text { a.e. } K \text {. }
$$

Thus,

$$
\frac{u^{\varepsilon_{j}}}{\varepsilon_{j}} \rightarrow-w_{0} \quad \text { a.e. } K
$$

Therefore,

$$
\int_{-w_{0}}^{\frac{u^{\varepsilon_{j}}}{\varepsilon_{j}}} f(s) \mathrm{d} s \rightarrow\left(\int_{-w_{0}}^{1} f(s) \mathrm{d} s\right) \mathcal{X}_{\{u>0\}} \quad \text { a.e. } \mathcal{D} \text {. }
$$


By using Proposition 2.3, Lemma 3.1 and (3.2) we can pass to the limit (for the sequence $\varepsilon_{j} \rightarrow$ 0 ) in the latter equation and get

$$
\begin{aligned}
& \iint_{\mathcal{D}} u_{t} u_{x_{1}} \psi=\frac{1}{2} \iint_{\mathcal{D}}|\nabla u|^{2} \psi_{x_{1}}-\iint_{\mathcal{D}} u_{x_{1}} \nabla u \nabla \psi+\iint_{\{u>0\}} M(x, t) \psi_{x_{1}} \\
& \quad+\iint_{\{u>0\}}\left(w_{0}\right)_{x_{1}}\left(\int_{-w_{0}}^{1} f(s) \mathrm{d} s\right) \psi
\end{aligned}
$$

for every $\psi \in C_{c}^{\infty}(\mathcal{D})$.

Now, let $\psi^{\lambda}(x, t)=\lambda \psi\left(\frac{x-x_{0}}{\lambda}, \frac{t-t_{0}}{\lambda^{2}}\right)$. Replacing $\psi$ by $\psi^{\lambda}$ in (3.3) and changing variables, we get, for $u_{\lambda}(x, t)=\frac{1}{\lambda} u\left(x_{0}+\lambda x, t_{0}+\lambda^{2} t\right)$,

$$
\begin{aligned}
& \iint\left(u_{\lambda}\right)_{t}\left(u_{\lambda}\right)_{x_{1}} \psi=\frac{1}{2} \iint\left|\nabla u_{\lambda}\right|^{2} \psi_{x_{1}}-\iint\left(u_{\lambda}\right)_{x_{1}} \nabla u_{\lambda} \nabla \psi \\
& \quad+\iint_{\left\{u_{\lambda}>0\right\}} M\left(\lambda x, \lambda^{2} t\right) \psi_{x_{1}}+\iint_{\{u>0\}}\left(w_{0}\right)_{x_{1}}\left(\int_{-w_{0}}^{1} f(s) \mathrm{d} s\right) \psi^{\lambda} .
\end{aligned}
$$

Let $r>0$ be such that $Q_{r}\left(x_{0}, t_{0}\right) \subset \subset \mathcal{D}$. We have that $u_{\lambda} \in \operatorname{Lip}\left(1, \frac{1}{2}\right)$ in $Q_{r / \lambda}(0,0)$ uniformly in $\lambda$, and $u_{\lambda}(0,0)=0$. Therefore, for every $\lambda_{n} \rightarrow 0$, there exists a subsequence $\lambda_{n^{\prime}} \rightarrow 0$ and a function $U \in \operatorname{Lip}\left(1, \frac{1}{2}\right)$ in $\mathbb{R}^{N+1}$ such that $u_{\lambda_{n^{\prime}}} \rightarrow U$ uniformly on compact sets of $\mathbb{R}^{N+1}$.

By our assumption on $\left(x_{0}, t_{0}\right)$, we can easily see that for every $k>0$

$$
\left|\left\{u_{\lambda}>0\right\} \cap\left\{x_{1}<0\right\} \cap Q_{k}(0,0)\right| \rightarrow 0 \quad \text { as } \quad \lambda \rightarrow 0,
$$

and

$$
\left|\left\{u_{\lambda}=0\right\} \cap\left\{x_{1}>0\right\} \cap Q_{k}(0,0)\right| \rightarrow 0 \quad \text { as } \quad \lambda \rightarrow 0 .
$$

Now, using Lemma 2.1 and the fact that $\psi^{\lambda} \rightarrow 0$ uniformly in $\mathcal{D}$ and supp $\psi^{\lambda} \subset \operatorname{supp} \psi$, we can pass to the limit in (3.4) and get

$$
\begin{aligned}
& \iint_{\left\{x_{1}>0\right\}} U_{t} U_{x_{1}} \psi= \\
& \frac{1}{2} \iint_{\left\{x_{1}>0\right\}}|\nabla U|^{2} \psi_{x_{1}}-\iint_{\left\{x_{1}>0\right\}} U_{x_{1}} \nabla U \nabla \psi+M(0,0) \iint_{\left\{x_{1}>0\right\}} \psi_{x_{1}} .
\end{aligned}
$$

Our aim is to prove that $U=\alpha x_{1}^{+}$. First, by (3.5) and (3.6), we deduce that $U=0$ in $\left\{x_{1}<0\right\}$. On the other hand, $U$ is a solution to the heat equation in $\{U>0\} \subset\left\{x_{1}>0\right\}$. By Corollary A.1 in [5], for every $\bar{x}^{\prime} \in \mathbb{R}^{N-1}, \bar{t} \in \mathbb{R}$, there exists $\alpha \geqslant 0$ such that

$$
U(x, t)=\alpha x_{1}^{+}+o\left(\left|\left(x_{1}, x^{\prime}\right)-\left(0, \bar{x}^{\prime}\right)\right|+|t-\bar{t}|^{1 / 2}\right) \quad \text { in } \quad\left\{x_{1}>0\right\} \cap\{t<\bar{t}\} .
$$

Replacing the test function $\psi$ by $\Phi^{\lambda}(x, t)=\lambda \Phi\left(\frac{x_{1}}{\lambda}, \frac{x^{\prime}-\bar{x}^{\prime}}{\lambda}, \frac{t-\bar{t}}{\lambda^{2}}\right)$ with $\Phi \in C_{c}^{\infty}(\{t<0\})$ and proceeding as above, we get

$$
-\frac{\alpha^{2}}{2} \iint_{\left\{x_{1}>0\right\}} \Phi_{x_{1}}+M(0,0) \iint_{\left\{x_{1}>0\right\}} \Phi_{x_{1}}=0 .
$$


In order to pass to the limit for a sequence $\lambda_{n} \rightarrow 0$ we have used Lemma 2.1. (See [6], Theorem 3.1 for the details.)

Thus, $\alpha=\sqrt{2 M(0,0)}$.

In order to see that $U=\alpha x_{1}^{+}$we use Lemma 2.4. In fact, by Lemma 2.1, there exists a sequence $j_{n} \rightarrow \infty$ such that

$$
u^{\delta_{n}}:=\frac{1}{\lambda_{n}} u^{\varepsilon_{j_{n}}}\left(\lambda_{n} x, \lambda_{n}^{2} t\right) \rightarrow U(x, t)
$$

uniformly on compact subsets of $\mathbb{R}^{N+1}$. We recall that $\left(u^{\delta_{n}}, v^{\delta_{n}}\right)$ is a solution to (1.1) with $\varepsilon$ replaced by $\delta_{n}$. Moreover,

$$
\frac{w^{\delta_{n}}}{\delta_{n}}=\frac{w^{\varepsilon_{j_{n}}}\left(\lambda_{n} x, \lambda_{n}^{2} t\right)}{\varepsilon_{j_{n}}} \rightarrow w_{0}(0,0)
$$

uniformly on compact sets of $\mathbb{R}^{N+1}$.

In addition, $U \geqslant 0$ and $\partial\{U>0\} \neq \emptyset$. By Lemma 2.4 we have that $|\nabla U| \leqslant \alpha(=\sqrt{2 M(0,0)})$. Since $U \equiv 0$ in $\left\{x_{1}=0\right\}$ we deduce that

$$
U \leqslant \alpha x_{1} \quad \text { in } \quad\left\{x_{1}>0\right\} .
$$

By Hopf's Principle, we deduce that

$$
U=\alpha x_{1} \quad \text { in } \quad\left\{x_{1}>0\right\} .
$$

The theorem is proved.

REMARK 3.1 It is clear from the proof that the result is still true if we replace condition (3.1) by the following property: $\frac{u^{\varepsilon_{j}}}{\varepsilon_{j}} \rightarrow-w_{0}$ a.e. $\{u \equiv 0\}^{\circ}$.

\section{Viscosity solutions}

In this section we prove that, under suitable assumptions, the limit function $u$ is a viscosity solution of the free-boundary problem (1.4).

For the sake of completeness, we state here the definition of viscosity solution that was introduced in [6] for the two-phase case of this problem when $w_{0}=0$.

Definition 4.1 Let $Q$ be a cylinder in $\mathbb{R}^{N} \times(0, T)$ and let $v \in C(\bar{Q})$. Then $v$ is called a classical subsolution (supersolution) of (1.4) in $Q$ if $v \geqslant 0$ and

1. $\Delta v-v_{t} \geqslant 0(\leqslant 0) \quad$ in $\quad \Omega^{+} \equiv Q \cap\{v>0\}$;

2. $v \in C^{1}\left(\overline{\Omega^{+}}\right)$;

3. for any $(x, t) \in \partial \Omega^{+} \cap Q, \nabla v(x, t) \neq 0, \quad$ and $\quad|\nabla v(x, t)| \geqslant \sqrt{2 M} \quad(\leqslant \sqrt{2 M})$.

We say that $v$ is a classical solution in $Q$ if it is both a classical subsolution and a classical supersolution.

DEFINITION 4.2 Let $u$ be a continuous non-negative function in $Q ; u$ is called a viscosity subsolution (supersolution) of (1.4) in $Q$ if, for every subcylinder $Q^{\prime} \subset \subset Q$ and for every classical supersolution (subsolution) $v$ in $Q^{\prime}$,

$$
\begin{aligned}
& u \leqslant v \quad \text { on } \quad \partial_{p} Q^{\prime} \quad\left(u \geqslant v \quad \text { on } \quad \partial_{p} Q^{\prime}\right) \quad \text { and } \\
& v>0 \quad \text { on } \overline{\{u>0\}} \cap \partial_{p} Q^{\prime} \quad\left(u>0 \quad \text { on } \overline{\{v>0\}} \cap \partial_{p} Q^{\prime}\right)
\end{aligned}
$$


implies that $u \leqslant v(u \geqslant v)$ in $Q^{\prime}$.

The function $u$ is called a viscosity solution if it is both a viscosity subsolution and a viscosity supersolution.

DEFINITION 4.3 Let $u$ be a continuous non-negative function in $\mathcal{D}$ and let $\left(x_{0}, t_{0}\right) \in \partial\{u>0\} \cap \mathcal{D}$. We say that $\left(x_{0}, t_{0}\right)$ is a regular point from the non-positive side, if there exists a regular non-negative function $v$ in $\mathcal{D}$ such that $v>u$ in $\{u>0\}$ for $t<t_{0}$ and $v\left(x_{0}, t_{0}\right)=u\left(x_{0}, t_{0}\right)$.

Finally we need the following definition on non-degeneracy.

DEFINITION 4.4 Let $u$ be a continuous non-negative function in $\mathcal{D}$. Let $\left(x_{0}, t_{0}\right) \in \mathcal{D}$ be such that $u\left(x_{0}, t_{0}\right)=0$. We say that $u$ does not degenerate at $\left(x_{0}, t_{0}\right)$ if there exist $r_{0}>0$ and $C>0$ such that

$$
\sup _{\partial_{p} Q_{r}^{-}\left(x_{0}, t_{0}\right)} u \geqslant C r \quad \text { for } 0<r \leqslant r_{0} .
$$

We now prove that, under suitable assumptions on the limit function $u$, it holds that $u$ is a viscosity solution to the free-boundary problem.

THEOREM 4.1 Let $u=\lim u^{\varepsilon_{k}}$, where $\left(u^{\varepsilon_{k}}, v^{\varepsilon_{k}}\right)$ are uniformly bounded solutions to (1.1) with $v^{\varepsilon_{k}} \geqslant 0$, satisfying (1.7) in $\mathcal{D}$, with $w_{0}>-1$, and such that $u^{\varepsilon_{k}}$ either satisfies (3.1) or $u_{t}^{\varepsilon_{k}} \leqslant 0$ in $\mathcal{D}$.

If $u^{+}$does not degenerate at every point of the free boundary which is regular from the nonpositive side, then $u$ is a viscosity solution of (1.4).

Proof. By Proposition 2.3 and Lemma 2.1, Theorem 4.1 of [6] can be stated for our system, thus $u$ is a viscosity supersolution.

In order to see that it is a viscosity subsolution, let $v$ be a classical supersolution such that

$$
u \leqslant v \quad \text { in } \quad \partial_{p} Q \quad \text { and } \quad v>0 \quad \text { in } \overline{\{u>0\}} \cap \partial_{p} Q .
$$

We want to see that $u \leqslant v$ in $Q$.

If it is not, we define

$$
t_{0}=\sup \{0<s<T: v>0 \quad \text { in } \quad \overline{\{u>0\}} \cap Q \cap\{0 \leqslant t<s\}\} .
$$

From the definition of $t_{0}$, it follows that $t_{0}>0$ and, from our hypotheses, we deduce that $v \geqslant u$ in $Q \cap\left\{0 \leqslant t<t_{0}\right\}$. In addition, there exists a sequence $(x(s), t(s)) \rightarrow\left(x_{0}, t_{0}\right) \in \bar{Q}$ such that $v(x(s), t(s))=0,(x(s), t(s)) \in \overline{\{u>0\}} \cap Q$. Clearly, $u\left(x_{0}, t_{0}\right)=v\left(x_{0}, t_{0}\right)=0$ and $\left(x_{0}, t_{0}\right) \in \partial\{u>0\} \cap Q$. If $\left(x_{0}, t_{0}\right) \in\{v=0\}^{\circ}$ then, for $\tau$ small, we have $u \leqslant v=0$ in $B_{\tau}\left(x_{0}, t_{0}\right) \cap\left\{t<t_{0}\right\}$ and, therefore, $u \equiv 0$ there, which contradicts our hypothesis. Thus,

$$
\begin{gathered}
v \geqslant u \quad \text { in } \quad Q \cap\left\{0 \leqslant t \leqslant t_{0}\right\}, \\
\left(x_{0}, t_{0}\right) \in \partial\{u>0\} \cap \partial\{v>0\} \cap Q .
\end{gathered}
$$

We may assume, without loss of generality, that $\left(x_{0}, t_{0}\right)=(0,0)$ and $Q_{1}(0,0)=Q_{1} \subset Q$ (consider instead of $u$ the function $\frac{1}{\lambda_{0}} u\left(x_{0}+\lambda_{0} x, t_{0}+\lambda_{0}^{2} t\right)$ for certain $\lambda_{0}>0$ small, and analogously with $v$ ). Let us take

$$
v_{\lambda}(x, t)=\frac{1}{\lambda} v\left(\lambda x, \lambda^{2} t\right), \quad u_{\lambda}(x, t)=\frac{1}{\lambda} u\left(\lambda x, \lambda^{2} t\right) .
$$


It is easy to see that there exists a sequence $\lambda_{n} \rightarrow 0$ and functions $u_{0}, v_{0}$ such that $v_{\lambda} \rightarrow$ $v_{0}, u_{\lambda} \rightarrow u_{0}$.

Since $v$ is regular, we have that $v_{0}(x, t)=\beta x_{1}^{+}$with $0 \leqslant \beta \leqslant \sqrt{2 M(0,0)}$ (for some system of coordinates).

Let us show also that $u_{0}(x, t)=\alpha x_{1}^{+}$for some $\alpha \geqslant 0$.

We may think that in $Q_{1}, \partial\{v>0\}$ is the graph of some function $\psi\left(x^{\prime}, t\right)=x_{1}, x=\left(x_{1}, x^{\prime}\right)$ with $\psi \in \operatorname{Lip}\left(1, \frac{1}{2}\right)$, where $\psi(0,0)=0$ and $\{v>0\}=\left\{x_{1}>\psi\left(x^{\prime}, t\right)\right\}$.

Hence, we have that

$$
\left|\psi\left(x^{\prime}, t\right)\right| \leqslant C\left(\left|x^{\prime}\right|+|t|^{1 / 2}\right) .
$$

Let $\mathcal{R}=\left\{(x, t) \in Q_{1}: x_{1}<-C\left(\left|x^{\prime}\right|+|t|^{1 / 2}\right)\right\}$. Then $\mathcal{R} \cap\{v>0\}=\emptyset$ and let $w$ be the caloric function in $\mathcal{O}=Q_{1}^{-} \backslash \mathcal{R}$ with $w=0$ in $\partial_{p} \mathcal{R}$ and $w=L \geqslant\|u\|_{\infty}$ in the rest of $\partial_{p} \mathcal{O}$.

Since $u$ is globally subcaloric and $u \leqslant w$ on $\partial_{p} \mathcal{O}$, then $u<w$ in $\mathcal{O}$.

Now, since $w-u$ is supercaloric in $\mathcal{O}, w-u>0$ in the interior and $w-u=0$ at $(0,0)$, then, by Lemma A.1 of [5], we have that $w-u=\delta x_{1}^{+}+o\left(|x|+|t|^{1 / 2}\right)$ and, since by the same lemma, $w$ has an asymptotic development at $(0,0)$,

$$
u(x, t)=\alpha x_{1}^{+}+o\left(|x|+|t|^{1 / 2}\right), \quad \text { with } \quad \alpha \geqslant 0 .
$$

Since by hypothesis $u^{+}$does not degenerate, it follows that $\alpha>0$.

On the other hand, since $v$ is regular, $v$ admits an asymptotic development at the origin in the form $v(x, t)=\beta x_{1}^{+}+o\left(|x|+|t|^{1 / 2}\right)$. Clearly, $\beta \geqslant \alpha$.

Now, let $h$ be the caloric function in $\tilde{\mathcal{O}}:=Q_{1}^{-} \cap\{v>0\} \cap\{-\mu<t<0\}$ for some small $\mu>0$, with $h=v-u$ on $\partial_{p} \tilde{\mathcal{O}}$. And, let $g$ be the caloric function in $\tilde{\mathcal{O}}$ with $g=v$ on $\partial_{p} \tilde{\mathcal{O}}$. Then, $h=g=0$ in $Q_{1}^{-} \cap \partial\{v>0\} \cap\{-\mu<t<0\}$ and $h>0, g>0$ in $\tilde{\mathcal{O}}$.

Therefore, by [1], there exists $\sigma>0$ such that $h \geqslant \sigma g$ in $Q_{1 / 2}^{-} \cap\{v>0\} \cap\left\{-\frac{\mu}{2}<t<0\right\}$.

Since $u$ is subcaloric in $Q_{1}^{-}$and $u \leqslant v$ in $Q_{1}^{-}$we deduce that $v-u \geqslant \sigma u$ in $Q_{1 / 2}^{-} \cap\{v>$ $0\} \cap\left\{-\frac{\mu}{2}<t<0\right\}$. In particular, $\beta-\alpha \geqslant \sigma \alpha>0$.

The theorem will be finished if we show that $\alpha=\sqrt{2 M(0,0)}$.

Case $1 \quad u^{\varepsilon_{k}}$ verifies (3.1).

As in Theorem 3.1, we obtain

$$
\begin{aligned}
& \iint_{\mathcal{D}} u_{t} u_{x_{1}} \psi=\frac{1}{2} \iint_{\mathcal{D}}|\nabla u|^{2} \psi_{x_{1}}-\iint_{\mathcal{D}} u_{x_{1}} \nabla u \nabla \psi \\
& \quad+\iint_{\mathcal{D} \cap\{u>0\}} M(x, t) \psi_{x_{1}}+\iint_{\mathcal{D} \cap\{u>0\}}\left(w_{0}\right)_{x_{1}}\left(\int_{-w_{0}}^{1} f(s) \mathrm{d} s\right) \psi
\end{aligned}
$$

for every test function $\psi$. Then, taking $\psi^{\lambda}(x, t)=\lambda \psi\left(\frac{x}{\lambda}, \frac{t}{\lambda^{2}}\right)$ and changing variables, we get

$$
\begin{aligned}
& \iint_{\mathcal{D}}\left(u_{\lambda}\right)_{t}\left(u_{\lambda}\right)_{x_{1}} \psi=\frac{1}{2} \iint_{\mathcal{D}}\left|\nabla u_{\lambda}\right|^{2} \psi_{x_{1}}-\iint_{\mathcal{D}}\left(u_{\lambda}\right)_{x_{1}} \nabla u_{\lambda} \nabla \psi \\
& \quad+\iint_{\mathcal{D} \cap\left\{u_{\lambda}>0\right\}} M\left(\lambda x, \lambda^{2} t\right) \psi_{x_{1}}+\iint_{\mathcal{D} \cap\{u>0\}}\left(w_{0}\right)_{x_{1}}\left(\int_{-w_{0}}^{1} f(s) \mathrm{d} s\right) \psi^{\lambda} .
\end{aligned}
$$


By Lemma 2.1, we get (for some sequence $\lambda_{k} \rightarrow 0$ )

$$
0=-\frac{1}{2} \alpha^{2} \iint_{\mathcal{D} \cap\left\{x_{1}>0\right\}} \psi_{x_{1}}+\lim _{k \rightarrow \infty} \iint_{\mathcal{D} \cap\left\{u_{\lambda_{k}}>0\right\}} M\left(\lambda_{k} x, \lambda_{k}^{2} t\right) \psi_{x_{1}} .
$$

We want to check that $\mathcal{X}_{\left\{u_{\lambda_{k}}>0\right\}} \rightarrow \mathcal{X}_{\left\{x_{1}>0\right\}}$ a.e. or, equivalently,

1. $\left\{x_{1}>0\right\} \subset \cup_{n=1}^{\infty} \cap_{k} \geqslant n\left\{u_{\lambda_{k}}>0\right\}=\liminf \left\{u_{\lambda_{k}}>0\right\}$ a.e.

2. $\cap_{n=1}^{\infty} \cup_{k \geqslant n}\left\{u_{\lambda_{k}}>0\right\}=\lim \sup \left\{u_{\lambda_{k}}>0\right\} \subset\left\{x_{1}>0\right\}$ a.e.

Let us show (1). If $x_{1}>0$, we get that $\alpha x_{1}>0$ and since $u_{\lambda_{k}}(x, t) \rightarrow \alpha x_{1}$ it follows that $u_{\lambda_{k}}(x, t)>0 \quad \forall k \geqslant k_{0}$.

Let us show (2). If there exists $k_{j} \rightarrow \infty$ with $u_{\lambda_{k}}(x, t)>0$ then it must be $x_{1} \geqslant 0$, because if $x_{1}<0$, we have that $v_{\lambda_{j}}(x, t)=0$ for $j \geqslant j_{0}$ (because as $v$ is regular, $\left\{v_{\lambda_{k}}>0\right\} \rightarrow\left\{x_{1}>0\right\}$ ). Since $u_{\lambda_{k_{j}}} \leqslant v_{\lambda_{k_{j}}}$ we get a contradiction.

Therefore,

$$
0=-\frac{1}{2} \alpha^{2} \iint_{\mathcal{D} \cap\left\{x_{1}>0\right\}} \psi_{x_{1}}+M(0,0) \iint_{\mathcal{D} \cap\left\{x_{1}>0\right\}} \psi_{x_{1}} .
$$

So that,

$$
0=\int_{\mathcal{D} \cap\left\{x_{1}=0\right\}}\left(\frac{1}{2} \alpha^{2}-M(0,0)\right) \psi \mathrm{d} x^{\prime} \mathrm{d} t .
$$

Since $\psi$ is arbitrary, $\frac{1}{2} \alpha^{2}=M(0,0)$, so that,

$$
\alpha=\sqrt{2 M(0,0)}
$$

and the proof is complete.

Case $2 u_{t}^{\varepsilon_{k}} \leqslant 0$.

We already know that, if we consider $u_{\lambda}(x, t)=\frac{1}{\lambda} u\left(\lambda x, \lambda^{2} t\right)$, then it follows that

$$
u_{\lambda}(x, t) \rightarrow u_{0}(x, t) \equiv \alpha x_{1}^{+}
$$

uniformly on compact subsets of $\mathbb{R}^{N+1}$.

As before,

$$
\begin{aligned}
& \iint_{\mathcal{D}} u_{t}^{\varepsilon_{k}} u_{x_{1}}^{\varepsilon_{k}} \psi=\frac{1}{2} \iint_{\mathcal{D}}\left|\nabla u^{\varepsilon_{k}}\right|^{2} \psi_{x_{1}}-\iint_{\mathcal{D}} u_{x_{1}}^{\varepsilon_{k}} \nabla u^{\varepsilon_{k}} \nabla \psi+\iint_{\mathcal{D}} \mathcal{B}_{\varepsilon_{k}}\left(u^{\varepsilon_{k}}, x, t\right) \psi_{x_{1}} \\
& \quad+\iint_{\mathcal{D}} w_{x_{1}}^{\varepsilon_{k}}\left(\int_{-w_{0} \varepsilon_{k}}^{u^{\varepsilon_{k}}} f_{\varepsilon_{k}}(s) \mathrm{d} s\right) \psi+\iint_{\mathcal{D}}\left(w_{0}\right)_{x_{1}}\left(\frac{w^{\varepsilon_{k}}}{\varepsilon_{k}}-w_{0}\right) f\left(-w_{0}\right) \psi .
\end{aligned}
$$

Now, as in the previous case, if we consider first $\psi^{\lambda}(x, t)=\lambda \psi\left(\frac{x}{\lambda}, \frac{t}{\lambda^{2}}\right)$ and change variables, we obtain

$$
\begin{aligned}
& \iint\left(u_{\lambda}^{\varepsilon_{k}}\right)_{t}\left(u_{\lambda}^{\varepsilon_{k}}\right)_{x_{1}} \psi=\frac{1}{2} \iint\left|\nabla u_{\lambda}^{\varepsilon_{k}}\right|^{2} \psi_{x_{1}}-\iint\left(u_{\lambda}^{\varepsilon_{k}}\right)_{x_{1}} \nabla u_{\lambda}^{\varepsilon_{k}} \nabla \psi \\
& \quad+\iint \mathcal{B}_{\varepsilon_{k} / \lambda}^{\lambda}\left(u_{\lambda}^{\varepsilon_{k}}, x, t\right) \psi_{x_{1}}+\iint_{\mathcal{D}} \frac{\left(w^{\varepsilon_{k}}\right)_{x_{1}}}{\varepsilon_{k}}\left(\int_{-w_{0}}^{\frac{u_{k}^{\varepsilon}}{\varepsilon_{k}}} f(s) \mathrm{d} s\right) \psi^{\lambda} \\
& \quad+\iint_{\mathcal{D}}\left(w_{0}\right)_{x_{1}}\left(\frac{w^{\varepsilon_{k}}}{\varepsilon_{k}}-w_{0}\right) f\left(-w_{0}\right) \psi^{\lambda}
\end{aligned}
$$


where $\mathcal{B}_{\varepsilon}^{\lambda}(u, x, t)=\int_{-w_{0}\left(\lambda x, \lambda^{2} t\right) \varepsilon}^{u}\left(s+w^{\varepsilon}(x, t)\right) f_{\varepsilon}(s) \mathrm{d} s$. We want to pass to the limit as both $\varepsilon_{k}$ and $\lambda$ go to zero.

Using Lemma 2.1, we see that for every sequence $\lambda_{n} \rightarrow 0$ there exists a sequence $k_{n} \rightarrow \infty$ such that $\delta_{n}:=\varepsilon_{k_{n}} / \lambda_{n} \rightarrow 0$ and $u^{\delta_{n}}:=\left(u^{\varepsilon_{k_{n}}}\right)_{\lambda_{n}} \rightarrow u_{0}$ uniformly on compact sets of $\mathbb{R}^{N+1}$. By Proposition 2.3 we see that we can pass to the limit in the first three terms of (4.1) (with $\varepsilon=\varepsilon_{k_{n}}$ and $\lambda=\lambda_{n}$ ).

Let us study the limit of $\mathcal{B}_{\delta_{n}}^{\lambda_{n}}\left(u^{\delta_{n}}(x, t), x, t\right)$.

It is easy to see that in $\left\{x_{1}>0\right\}, \mathcal{B}_{\delta_{n}}^{\lambda_{n}}\left(u^{\delta_{n}}(x, t), x, t\right) \rightarrow M(0,0)$ uniformly on compact sets. Now, let $K \subset\left\{x_{1}<0\right\}$ be compact. We will show that

$$
\nabla\left(\mathcal{B}_{\delta_{n}}^{\lambda_{n}}\left(u^{\delta_{n}}(x, t), x, t\right)\right) \rightarrow 0 \quad \text { in } \quad L^{1}(K) .
$$

In fact,

$$
\begin{aligned}
& \nabla\left(\mathcal{B}_{\delta_{n}}^{\lambda_{n}}\left(u^{\delta_{n}}(x, t), x, t\right)\right)=v^{\delta_{n}} f_{\delta_{n}}\left(u^{\delta_{n}}\right) \nabla u^{\delta_{n}} \\
& +\lambda_{n} \nabla w_{0}\left(\lambda_{n} x, \lambda_{n}^{2} t\right)\left(\frac{w^{\delta_{n}}}{\delta_{n}}(x, t)-w_{0}\left(\lambda_{n} x, \lambda_{n}^{2} t\right)\right) f\left(-w_{0}\left(\lambda_{n} x, \lambda_{n}^{2} t\right)\right) \\
& \quad+\frac{\nabla w^{\delta_{n}}}{\delta_{n}} \int_{-w_{0}\left(\lambda_{n} x, \lambda_{n}^{2} t\right)}^{\frac{u^{\delta_{n}}}{\delta_{n}}} f(s) \mathrm{d} s .
\end{aligned}
$$

Since $v^{\delta_{n}} f_{\delta_{n}}\left(u^{\delta_{n}}\right) \rightarrow 0$ as measures in $K$ and is non-negative, we deduce that the convergence takes place in $L^{1}(K)$. On the other hand, $\nabla u^{\delta_{n}}$ is uniformly bounded. Therefore, the first term goes to zero in $L^{1}(K)$.

In order to see that the second and third terms go to zero uniformly in $K$ we only need to observe that

$$
\frac{u^{\delta_{n}}}{\delta_{n}}(x, t)=\frac{u^{\varepsilon_{k_{n}}}}{\varepsilon_{k_{n}}}\left(\lambda_{n} x, \lambda_{n}^{2} t\right)
$$

and a similar formula holds for $\frac{w^{\delta_{n}}}{\delta_{n}}$. So that

$$
\begin{aligned}
& \left|\frac{w^{\delta_{n}}}{\delta_{n}}(x, t)-w_{0}\left(\lambda_{n} x, \lambda_{n}^{2} t\right)\right| \rightarrow 0 \quad \text { uniformly on compact sets of } \mathbb{R}^{N+1}, \\
& \frac{u^{\delta_{n}}}{\delta_{n}} \geqslant-\frac{w^{\delta_{n}}}{\delta_{n}} \geqslant-C, \\
& \frac{\left|\nabla w^{\delta_{n}}\right|}{\delta_{n}}(x, t)=\lambda_{n} \frac{\left|\nabla w^{\varepsilon_{k_{n}}}\right|}{\varepsilon_{k_{n}}}\left(\lambda_{n} x, \lambda_{n}^{2} t\right) \rightarrow 0 \quad \text { uniformly on compact sets of } \mathbb{R}^{N+1} .
\end{aligned}
$$

On the other hand, $\left|\mathcal{B}_{\delta_{n}}^{\lambda_{n}}\left(u^{\delta_{n}}(x, t), x, t\right)\right| \leqslant C_{K}$, so that we have

$$
\mathcal{B}_{\delta_{n}}^{\lambda_{n}}\left(u^{\delta_{n}}(x, t), x, t\right) \rightarrow \bar{M}(t) \quad \text { weakly in } \quad L^{2}(K) .
$$

Let us now show that, actually, the convergence takes place in $L^{1}(K)$.

It holds that

$$
\begin{gathered}
\frac{\partial}{\partial t}\left(\mathcal{B}_{\delta_{n}}^{\lambda_{n}}\left(u^{\delta_{n}}(x, t), x, t\right)\right)=v^{\delta_{n}} f_{\delta_{n}}\left(u^{\delta_{n}}\right)\left(u^{\delta_{n}}\right)_{t}+\frac{\partial}{\partial t} \mathcal{B}_{\delta_{n}}^{\lambda_{n}}\left(u^{\delta_{n}}, x, t\right) \\
\leqslant \frac{\partial}{\partial t} \mathcal{B}_{\delta_{n}}^{\lambda_{n}}\left(u^{\delta_{n}}, x, t\right) \leqslant C_{K} \quad \text { in } \quad K .
\end{gathered}
$$


On the other hand, for every $\left(x_{0}, t_{0}\right) \in K$, and $Q_{\tau}\left(x_{0}, t_{0}\right) \subset\left\{x_{1}<0\right\}$,

$$
\begin{gathered}
\iint_{Q_{\tau}\left(x_{0}, t_{0}\right)} \frac{\partial}{\partial t}\left(\mathcal{B}_{\delta_{n}}^{\lambda_{n}}\left(u^{\delta_{n}}(x, t), x, t\right)\right)=\int_{B_{\tau}\left(x_{0}\right)} \mathcal{B}_{\delta_{n}}^{\lambda_{n}}\left(u^{\delta_{n}}\left(x, t_{0}+\tau^{2}\right), x,\left(t_{0}+\tau^{2}\right)\right) \mathrm{d} x \\
-\int_{B_{\tau}\left(x_{0}\right)} \mathcal{B}_{\delta_{n}}^{\lambda_{n}}\left(u^{\delta_{n}}\left(x, t_{0}-\tau^{2}\right), x,\left(t_{0}-\tau^{2}\right)\right) \mathrm{d} x \\
\geqslant-C_{\tau}
\end{gathered}
$$

since $\left|\mathcal{B}_{\delta_{n}}^{\lambda_{n}}\left(u^{\delta_{n}}(x, t), x, t\right)\right| \leqslant C_{K}$ for every compact set $K$.

Therefore, there exists $C_{K}>0$ such that $\left\|\mathcal{B}_{\delta_{n}}^{\lambda_{n}}\left(u^{\delta_{n}}(x, t), x, t\right)\right\|_{W^{1,1}(K)} \leqslant C_{K}$. Hence the convergence takes place in $L^{1}(K)$ (for a subsequence).

Now arguing as in Lemma 3.1, we get $\bar{M}(t)=0$ or $\bar{M}(t)=M(0,0)$.

We can now take the limit in (4.1) for the sequences $\varepsilon_{k_{n}}$ and $\lambda_{n}$ and we obtain

$$
0=-\frac{1}{2} \alpha^{2} \iint_{\mathcal{D} \cap\left\{x_{1}>0\right\}} \psi_{x_{1}}+M(0,0) \iint_{\mathcal{D} \cap\left\{x_{1}>0\right\}} \psi_{x_{1}}+\iint_{\mathcal{D} \cap\left\{x_{1}<0\right\}} \bar{M}(t) \psi_{x_{1}} .
$$

Thus,

$$
0=\int_{\mathcal{D} \cap\left\{x_{1}=0\right\}}\left(\frac{1}{2} \alpha^{2}-M(0,0)-\bar{M}(t)\right) \psi \mathrm{d} x^{\prime} \mathrm{d} t .
$$

Since $\psi$ is arbitrary we get $\frac{1}{2} \alpha^{2}=M(0,0)-\bar{M}(t)$. So that, in particular, $\bar{M}(t)$ is constant and then we have that $\bar{M}(t) \equiv 0$ or $\bar{M}(t) \equiv M(0,0)$. Since $\alpha>0$ we deduce that $\bar{M}(t) \equiv 0$ and

$$
\alpha=\sqrt{2 M(0,0)} .
$$

The proof is complete.

Now we prove a proposition that says that, under suitable assumptions, $u^{+}$does not degenerate on the free boundary. The proof is similar to Theorem 6.3 in [5], where the non-degeneracy of $u^{+}$ was proved in the strictly two- phase case. Here we assume, instead of (3.1), the somewhat stronger condition that for every $K \subset \mathcal{D}$ compact, there exist $0<\eta<1$ and $\varepsilon_{0}>0$ such that for every $0<\varepsilon \leqslant \varepsilon_{0}$

$$
\frac{u^{\varepsilon}}{\varepsilon} \leqslant \eta \quad \text { in } \quad K \cap\{u \equiv 0\}^{\circ} .
$$

Proposition 4.1 Let $u=\lim u^{\varepsilon_{k}}$, where $\left(u^{\varepsilon_{k}}, v^{\varepsilon_{k}}\right)$ are uniformly bounded solutions to (1.1) satisfying (1.7) with $w_{0}>-1$, such that $v^{\varepsilon_{k}} \geqslant 0$ and the functions $u^{\varepsilon_{k}}$ satisfy (4.2). Let $\left(x_{0}, t_{0}\right) \in$ $\partial\{u>0\}$.

Let us assume that there exists $v \in \mathbb{R}^{N}$, with $|v|=1$ such that

$$
\liminf _{r \rightarrow 0^{+}} \frac{\left|\{u>0\} \cap\left\{\left\langle x-x_{0}, v\right\rangle>0\right\} \cap Q_{r}^{-}\left(x_{0}, t_{0}\right)\right|}{\left|Q_{r}^{-}\left(x_{0}, t_{0}\right)\right|}>\alpha_{1}
$$

and

$$
\liminf _{r \rightarrow 0^{+}} \frac{\left|\{u=0\}^{\circ} \cap\left\{\left\langle x-x_{0}, \nu\right\rangle<0\right\} \cap Q_{r}^{-}\left(x_{0}, t_{0}\right)\right|}{\left|Q_{r}^{-}\left(x_{0}, t_{0}\right)\right|}>\alpha_{2}
$$


with $\alpha_{1}+\alpha_{2}>\frac{1}{2}$, then there exists a constant $C>0$ and $r_{0}>0$ which depends on $N$ and $f$ such that, if $0<r \leqslant r_{0}$,

$$
\sup _{\partial_{p} Q_{r}^{-}\left(x_{0}, t_{0}\right)} u \geqslant C r .
$$

Proof. Without loss of generality, we may assume that $\left(x_{0}, t_{0}\right)=(0,0)$ and that $v=e_{1}=$ $(1,0, \ldots, 0)$.

Here we note $Q_{r}^{-}=Q_{r}^{-}(0,0)$ and

$$
\left(u^{\varepsilon}\right)_{r}(x, t)=\frac{1}{r} u^{\varepsilon}\left(r x, r^{2} t\right), \quad\left(v^{\varepsilon}\right)_{r}(x, t)=\frac{1}{r} v^{\varepsilon}\left(r x, r^{2} t\right), \quad u_{r}(x, t)=\frac{1}{r} u\left(r x, r^{2} t\right) .
$$

Let us see that there exists $r_{0}>0$ and a constant $c$ such that if $r<r_{0}$ and $\varepsilon<\varepsilon_{0}=\varepsilon_{0}(r)$, then

$$
\iint_{Q_{1}^{-}}\left(v^{\varepsilon}\right)_{r} f_{\varepsilon / r}\left(\left(u^{\varepsilon}\right)_{r}\right) \mathrm{d} x \geqslant c .
$$

Following [5], there exists $\gamma>0$ small such that, for some $\lambda>0$,

$$
\frac{\left|\left\{u_{r}>\gamma\right\} \cap\left\{x_{1}>0\right\} \cap Q_{1}^{-}\right|}{\left|Q_{1}^{-}\right|}+\frac{\left|\left\{u_{r}=0\right\}^{\circ} \cap\left\{x_{1}<0\right\} \cap Q_{1}^{-}\right|}{\left|Q_{1}^{-}\right|} \geqslant \frac{1}{2}+\lambda .
$$

Let us now define

$$
\begin{gathered}
A_{r}=\left\{u_{r}>\gamma\right\} \cap\left\{x_{1}>0\right\} \cap Q_{1}^{-}, \quad B_{r}=\left\{u_{r}=0\right\}^{\circ} \cap\left\{x_{1}<0\right\} \cap Q_{1}^{-} \\
\text {and }-B_{r}=\left\{\left(x_{1}, x^{\prime}, t\right) /\left(-x_{1}, x^{\prime}, t\right) \in B_{r}\right\} .
\end{gathered}
$$

Then, we have

$$
\left|A_{r} \cap\left(-B_{r}\right)\right| \geqslant \lambda\left|Q_{1}^{-}\right|=\tilde{\lambda} .
$$

Once again, following [5], we have for $0<\rho<1$ fixed that there exists $0<x_{1}^{r}<1$ such that

$$
\left|\Lambda_{r}\right|=\left|\left\{\left(x^{\prime}, t\right) /\left(x_{1}^{r}, x^{\prime}, t\right) \in A_{r} \cap\left(-B_{r}\right)\right\}\right|>\rho \tilde{\lambda} .
$$

Let $\eta>0$ be the constant in (4.2) in $Q_{1}(0,0)$ and let $0<\delta^{\prime}<\delta, 0<b<b^{\prime}<1$ be such that

$$
\eta<-w_{0}(0,0)+\delta<b .
$$

Let $\kappa>0$ be such that

$$
f(s)>\kappa>0 \quad \text { for } s \leqslant b^{\prime} .
$$

Then, for $\left(x^{\prime}, t\right) \in \Lambda_{r}$, we have (for $\varepsilon=\varepsilon_{\kappa}$ )

$$
\frac{1}{\varepsilon / r}\left(u^{\varepsilon}\right)_{r}\left(x_{1}^{r}, x^{\prime}, t\right)>\frac{\gamma}{2(\varepsilon / r)}>b, \quad \frac{1}{\varepsilon / r}\left(u^{\varepsilon}\right)_{r}\left(-x_{1}^{r}, x^{\prime}, t\right)<-w_{0}(0,0)+\delta
$$

if $\varepsilon_{k}<\varepsilon_{1}=\varepsilon_{1}(r)$ is small. So that, for every $\left(x^{\prime}, t\right) \in \Lambda_{r}$, there exists $\tilde{x}_{1}^{r} \in(-1,1)$ such that $-w_{0}(0,0)+\delta \leqslant \frac{1}{\varepsilon / r}\left(u^{\varepsilon}\right)_{r}\left(\tilde{x}_{1}^{r}, x^{\prime}, t\right) \leqslant b$. 
Now, by the uniform Lipschitz regularity of $\left(u^{\varepsilon}\right)_{r}$ and $\left(v^{\varepsilon}\right)_{r}$, and (1.7), we have that for $\varepsilon \leqslant$ $\varepsilon_{0}\left(\leqslant \varepsilon_{1}\right)$ and $r \leqslant r_{0}$,

$$
\frac{\left(u^{\varepsilon}\right)_{r}}{\varepsilon / r}\left(x_{1}, x^{\prime}, t\right) \leqslant b^{\prime} \quad \text { and } \quad \frac{\left(v^{\varepsilon}\right)_{r}}{\varepsilon / r}\left(x_{1}, x^{\prime}, t\right) \geqslant \delta^{\prime} \quad \text { if } \quad\left|x_{1}-\tilde{x}_{1}^{r}\right|<C \frac{\varepsilon}{r}
$$

where $C$ depends on $\delta, \delta^{\prime}, b, b^{\prime}$, on the Lipschitz constant of $u^{\varepsilon}$ and $v^{\varepsilon}$ in $Q_{1}^{-}$and $r_{0}$ depends only $w_{0}$.

Finally we have

$$
\begin{gathered}
\iint_{Q_{1}^{-}}\left(v^{\varepsilon}\right)_{r} f_{\varepsilon / r}\left(\left(u^{\varepsilon}\right)_{r}\right)=\iint_{Q_{1}^{-}} \frac{\left(v^{\varepsilon}\right)_{r}}{\varepsilon / r} \frac{1}{\varepsilon / r} f\left(\frac{\left(u^{\varepsilon}\right)_{r}}{\varepsilon / r}\right) \\
\geqslant \delta^{\prime} \frac{\kappa}{\varepsilon / r} \mid\left\{(x, t) \in Q_{1}^{-} / \frac{\left(v^{\varepsilon}\right)_{r}}{\varepsilon / r} \geqslant \delta^{\prime} \text { and } f\left(\frac{\left(u^{\varepsilon}\right)_{r}}{\varepsilon / r}\right) \geqslant \kappa\right\} \mid \\
\geqslant \delta^{\prime} \frac{\kappa}{\varepsilon / r}\left|\Lambda_{r}\right| 2 C \frac{\varepsilon}{r} \geqslant 2 C \delta^{\prime} \kappa \rho \tilde{\delta} \equiv c .
\end{gathered}
$$

The rest of the proof follows as in [5].

REMARK 4.1 Proposition 4.1 remains true if we change the hypothesis that $u^{\varepsilon_{k}}$ satisfies (4.2) by

$$
\frac{u^{\varepsilon_{k}}}{\varepsilon_{k}} \rightarrow-w_{0} \quad \text { a.e. }\{u \equiv 0\}^{\circ} .
$$

In fact, as in the proof of Proposition 4.1, we consider for each $0<r<1$ the sets $A_{r}$ and $B_{r}$. So that, for some $0<\lambda<1$

$$
\left|A_{r} \cap\left(-B_{r}\right)\right| \geqslant \lambda\left|Q_{1}^{-}\right| .
$$

Since $B_{r} \subset\left\{u_{r} \equiv 0\right\}^{\circ}$, it holds that

$$
\frac{\left(u^{\varepsilon}\right)_{r}}{\varepsilon / r}\left(-x_{1}, x^{\prime}, t\right) \rightarrow-w_{0}\left(-r x_{1}, r x^{\prime}, r^{2} t\right) \quad \text { a.e. } A_{r} \cap\left(-B_{r}\right) .
$$

Let $0<\mu<1$. There exists $C_{r} \subset\left(A_{r} \cap\left(-B_{r}\right)\right)$ such that $\left|C_{r}\right|=\mu\left|A_{r} \cap\left(-B_{r}\right)\right|$ and

$$
\frac{\left(u^{\varepsilon}\right)_{r}}{\varepsilon / r}\left(-x_{1}, x^{\prime}, t\right) \rightarrow-w_{0}\left(-r x_{1}, r x^{\prime}, r^{2} t\right) \quad \text { uniformly in } C_{r} \text {. }
$$

Let $\delta>0$. There exists $\varepsilon_{1}=\varepsilon_{1}(r)$ such that

$$
\frac{\left(u^{\varepsilon}\right)_{r}}{\varepsilon / r}\left(-x_{1}, x^{\prime}, t\right) \leqslant-w_{0}\left(-r x_{1}, r x^{\prime}, r^{2} t\right)+\frac{\delta}{2} \leqslant-w_{0}(0,0)+\delta \quad \text { in } \quad C_{r}
$$

if $\varepsilon<\varepsilon_{1}$ and $r<r_{0}=r_{0}(\delta)$. Now, the proof follows as in Proposition 4.1 by taking $\tilde{\lambda}=\mu \lambda\left|Q_{1}^{-}\right|$ and

$$
\Lambda_{r}:=\left\{\left(x^{\prime}, t\right) /\left(x_{1}^{r}, x^{\prime}, t\right) \in C_{r}\right\} .
$$

REMARK 4.2 Proposition 4.1 remains true if we change condition (4.2) by condition (3.1). In fact, as in the proof of Theorem 3.1, we see that condition (3.1) implies that

$$
\mathcal{B}_{\varepsilon_{k}}\left(u^{\varepsilon_{k}}, x, t\right) \rightarrow 0 \quad L_{\text {loc }}^{1}\left(\{u \equiv 0\}^{\circ}\right) .
$$

As in Theorem 3.1 we deduce that $u^{\varepsilon_{k}}$ satisfies (4.3). 
Using Remark 4.1, Remark 4.2 and Theorem 4.1 we get the following Corollaries.

COROLlaRY 4.1 Let $u=\lim u^{\varepsilon_{k}}$ where $\left(u^{\varepsilon_{k}}, v^{\varepsilon_{k}}\right)$ are unifomly bounded solutions to (1.1) in a domain $\mathcal{D} \subset \mathbb{R}^{N+1}$ with $v^{\varepsilon_{k}} \geqslant 0$, which verify (1.7) with $w_{0}>-1$ and such that $u^{\varepsilon_{k}}$ satisfies (3.1). If the free boundary $\mathcal{D} \cap \partial\{u>0\}$ is given by $x_{1}=g\left(x^{\prime}, t\right)$ with $g \in \operatorname{Lip}\left(1, \frac{1}{2}\right)$, then $u$ is a viscosity solution of the free-boundary problem (1.4).

COROLLARY 4.2 Let $u=\lim u^{\varepsilon_{k}}$ where $\left(u^{\varepsilon_{k}}, v^{\varepsilon_{k}}\right)$ are unifomly bounded solutions to (1.1) in a domain $\mathcal{D} \subset \mathbb{R}^{N+1}$ with $v^{\varepsilon_{k}} \geqslant 0$, which verify (1.7) with $w_{0}>-1$, and such that $u^{\varepsilon_{k}}$ satisfies (4.3) and $u_{t}^{\varepsilon_{k}} \leqslant 0$. If, for every $\left(x_{0}, t_{0}\right) \in \mathcal{D} \cap \partial\{u>0\},\left\{x \in \mathbb{R}^{N} /\left(x, t_{0}\right) \in \mathcal{D} \cap\{u>0\}\right\}$ is given by $x_{1}>\Phi\left(x^{\prime}\right)$ with $\Phi$ Lipschitz continuous then, $u$ is a viscosity solution of the free-boundary problem (1.4).

Proof. We only need to see that $u$ does not degenerate at points of the free boundary which are regular from the zero side. Let $\left(x_{0}, t_{0}\right)$ be any such point. We see that we can apply Remark 4.1 at that point. In fact, since $u_{t}^{\varepsilon_{k}} \leqslant 0, u$ is decreasing in time. Therefore,

$$
\left\{(x, t) / x_{1}>\Phi(x), t \leqslant t_{0}\right\} \subset\{u>0\}
$$

and the parabolic density of this set is positive.

In particular, Corollary 4.2 can be applied to solutions of (1.1) with $u_{0}^{\varepsilon}$ constructed as in [7] and $v_{0}^{\varepsilon}$ a small perturbation of $u_{0}^{\varepsilon}$.

COROLlaRY 4.3 Let $u_{0} \in C\left(\mathbb{R}^{N}\right) \cap C^{2}\left(\overline{\left\{u_{0}>0\right\}}\right)$ be such that $\left\|u_{0}\right\|_{C^{2}\left(\overline{\left\{u_{0}>0\right\}}\right)}<\infty, \Delta u_{0} \leqslant 0$ and $\left(u_{0}\right)_{x_{1}}-\lambda\left|\nabla u_{0}\right| \geqslant 0$ in $\left\{u_{0}>0\right\}$ with $\lambda>0$. Assume, moreover, that $0<a_{2} \leqslant\left|\nabla u_{0}\right| \leqslant$ $a_{1}<\sqrt{2 M_{0}}$ in a neighbourhood of the free boundary: $\left\{x \in\left\{u_{0}>0\right\} / \operatorname{dist}\left(x,\left\{u_{0}=0\right\}\right) \leqslant \gamma\right\}$, and $M_{0}=\int_{0}^{1} s f(s)$. Then, there exists a sequence $\left(u_{0}^{\varepsilon}, v_{0}^{\varepsilon}\right) \in\left(C^{1}\left(\mathbb{R}^{N}\right)\right)^{2}$ with $u_{0}^{\varepsilon} \rightarrow u_{0}$ uniformly in $\mathbb{R}^{N}$ (so that $u_{0}^{\varepsilon}$ are uniformly bounded) and, moreover, it satisfies

$$
\begin{aligned}
& \text { 1. } \Delta u_{0}^{\varepsilon}-v_{0}^{\varepsilon} f_{\varepsilon}\left(u_{0}^{\varepsilon}\right) \leqslant 0 \\
& \text { 2. }\left(u_{0}^{\varepsilon}\right)_{x_{1}}-\lambda\left|\nabla u_{0}^{\varepsilon}\right| \geqslant 0 \\
& \text { 3. } \frac{v_{0}^{\varepsilon}-u_{0}^{\varepsilon}}{\varepsilon} \rightarrow w_{0} \text { uniformly on compact sets, with } w_{0}>-1 \text {. }
\end{aligned}
$$

$w_{0} \in \mathbb{R}$ is any constant such that $w_{0} \geqslant-\eta$ with $\eta>0$ small enough.

Let $\left(u^{\varepsilon}, v^{\varepsilon}\right)$ be the solution to (1.1) with initial datum $\left(u_{0}^{\varepsilon}, v_{0}^{\varepsilon}\right)$ (so that, in particular, $u^{\varepsilon}$ and $v^{\varepsilon}$ are uniformly bounded). For every sequence $\varepsilon_{j} \rightarrow 0$ there exists a subsequence $\varepsilon_{j_{k}}$ such that there exists

$$
u=\lim _{k \rightarrow \infty} u^{\varepsilon_{j_{k}}}
$$

and $u$ is a viscosity solution to the free-boundary problem (1.4).

Proof. Let $u_{0}^{\varepsilon}$ be the approximations constructed in [7]. The approximations are constructed in the following way. First, we extend $u_{0}$ to a neighbourhood of $\left\{u_{0}>0\right\}: S:=\left\{x \in \mathbb{R}^{N} / \operatorname{dist}\left(x,\left\{u_{0}>\right.\right.\right.$ $0\}) \leqslant \gamma\}$ in such a way that $\left\|u_{0}\right\|_{C^{2}(S)}<\infty$. For $\varepsilon$ small enough we define

$$
u_{0}^{\varepsilon}(x)=\varepsilon F\left(\frac{1}{\sqrt{2 M_{0}}}\left(1-\frac{u_{0}(x)}{\varepsilon}\right)\right) \quad \text { in } \quad\left\{-C \varepsilon \leqslant u_{0} \leqslant \varepsilon\right\},
$$


where $F \in C^{2}(\mathbb{R})$ is such that

$$
F^{\prime \prime} \leqslant(1+\delta) F f(F)+\alpha F^{\prime}, \quad F(0)=1, \quad F^{\prime}(0)=-\sqrt{2 M_{0}} .
$$

Here $\delta>0, \alpha>0$ are such that $F$ has a strict minimum at a finite point $\bar{s}$ such that $\bar{s} \sqrt{2 M_{0}}>1$. $(\bar{s} \rightarrow+\infty$ as $\delta \rightarrow 0)$, and $F$ is decreasing for $s<\bar{s}$.

The constant $C$ is taken as $C=\bar{s} \sqrt{2 M_{0}}-1$.

We define

$$
\begin{array}{lll}
u_{0}^{\varepsilon}=u_{0} & \text { in } & \left\{u_{0}>\varepsilon\right\} \\
u_{0}^{\varepsilon}=\varepsilon F(\bar{s}) & \text { in } & \mathbb{R}^{N} \backslash\left\{u_{0}>-C \varepsilon\right\} .
\end{array}
$$

As in [7], we see that $u_{0}^{\varepsilon} \in C^{1}\left(\mathbb{R}^{N}\right)$.

Let $w_{0} \in \mathbb{R}$ be such that $w_{0} \geqslant-\eta>-F(\bar{s})$ with $\eta>0$ to be fixed later and let

$$
v_{0}^{\varepsilon}=u_{0}^{\varepsilon}+\varepsilon w_{0} .
$$

Then, $v_{0}^{\varepsilon} \geqslant 0$. It is straightforward to verify that $(4.4)_{1}$ is satisfied in $\left\{u_{0}>\varepsilon\right\}$ and $\mathbb{R}^{N} \backslash\left\{u_{0}>-C \varepsilon\right\}$. Let us see that it is satisfied in $\left\{-C \varepsilon \leqslant u_{0} \leqslant \varepsilon\right\}$. In fact,

$$
\begin{aligned}
\Delta u_{0}^{\varepsilon} & -v_{0}^{\varepsilon} f_{\varepsilon}\left(u_{0}^{\varepsilon}\right)=\frac{1}{2 M_{0} \varepsilon} F^{\prime \prime}\left|\nabla u_{0}\right|^{2}-\frac{1}{\sqrt{2 M_{0}}} F^{\prime} \Delta u_{0}-\frac{1}{\varepsilon} F f(F)-\frac{w_{0}}{\varepsilon} f(F) \\
& \leqslant \frac{1+\delta}{2 M_{0} \varepsilon} F f(F)\left|\nabla u_{0}\right|^{2}+\frac{\alpha}{2 M_{0} \varepsilon} F^{\prime}\left|\nabla u_{0}\right|^{2}-\frac{a}{\sqrt{2 M_{0}}} F^{\prime}-\frac{1}{\varepsilon} F f(F)-\frac{w_{0}}{\varepsilon} f(F)
\end{aligned}
$$

where $a>0$ is such that $\left|\Delta u_{0}\right| \leqslant a$.

Let $0<\mu<1$ be such that $a_{1} \leqslant(1-\mu)^{1 / 2} A \sqrt{2 M_{0}}$ with $0<A<1$, and let $\delta$ in the definition of $F$ be such that $(1+\delta) A^{2} \leqslant 1$. Then, if $\varepsilon$ is small enough so that $\alpha a_{2}^{2} / \sqrt{2 M_{0}}>a \varepsilon$ it holds that

$$
\begin{aligned}
\Delta u_{0}^{\varepsilon} & -v_{0}^{\varepsilon} f_{\varepsilon}\left(u_{0}^{\varepsilon}\right) \leqslant \frac{1}{\varepsilon}\left[\left[(1+\delta)(1-\mu) A^{2}-1\right] F f(F)+\left(\frac{\alpha a_{2}^{2}}{2 M_{0}}-\frac{a \varepsilon}{\sqrt{2 M_{0}}}\right) F^{\prime}-w_{0} f(F)\right] \\
& \leqslant \frac{1}{\varepsilon}\left[-\mu F-w_{0}\right] f(F) \leqslant \frac{1}{\varepsilon}\left[-\mu F(\bar{s})-w_{0}\right] f(F) \leqslant 0
\end{aligned}
$$

if $\eta=\mu F(\bar{s})$.

Clearly, $(4.4)_{3}$ holds. Let us see that $(4.4)_{2}$ also holds. We only need to verify this property in the set $\left\{-C \varepsilon<u_{0}<\varepsilon\right\}$ and this is clear from the fact that

$$
\nabla u_{0}^{\varepsilon}=-\frac{1}{\sqrt{2 M_{0}}} F^{\prime}\left(\frac{1}{\sqrt{2 M_{0}}}\left(1-\frac{u_{0}}{\varepsilon}\right)\right) \nabla u_{0} .
$$

Now, by the results of Section 2 , for every sequence $\varepsilon_{j} \rightarrow 0$ there exists a subsequence and a continuous function $u$ such that $u^{\varepsilon_{j_{k}}} \rightarrow u$ uniformly on compact subsets of $\mathbb{R}^{N} \times(0, \infty)$.

On the other hand, $u_{t}^{\varepsilon}$ is a solution to the following equation:

$$
\Delta U-U_{t}=\beta_{\varepsilon}^{\prime}(u) U .
$$

Here $\beta_{\varepsilon}(s)=s f_{\varepsilon}(s)$. Since, for $\varepsilon$ small enough, $u_{t}^{\varepsilon}(x, 0) \leqslant 0$ we conclude that

$$
u_{t}^{\varepsilon} \leqslant 0 \quad \text { in } \quad \mathbb{R}^{N} \times(0, \infty) .
$$


In a similar way, we see that $u_{x_{1}}^{\varepsilon}-\lambda u_{x_{i}}^{\varepsilon} \geqslant 0$ for every $i$. So that

$$
u_{x_{1}}^{\varepsilon}-\frac{\lambda}{N}\left|\nabla u^{\varepsilon}\right| \geqslant 0 \quad \text { in } \quad \mathbb{R}^{N} \times(0, \infty) .
$$

Clearly (4.5) and (4.6) imply that

$$
u_{t} \leqslant 0 \quad \text { and } \quad u_{x_{1}}-\frac{\lambda}{N}|\nabla u| \geqslant 0 \quad \text { in } \quad\{u>0\} .
$$

In particular, the free boundary is Lipschitz in space.

So that, in order to apply Corollary 4.2, we only need to verify that $u^{\varepsilon_{k}}$ satisfies (4.3). On one hand, given $K \subset\left\{u_{0} \equiv 0\right\}^{\circ}$ compact, there exists $\varepsilon_{0}$ such that for $\varepsilon<\varepsilon_{0}$

$$
\mathcal{B}_{\varepsilon}\left(u_{0}^{\varepsilon}, x, 0\right)=\int_{-w_{0}}^{\frac{u_{0}^{\varepsilon}}{\varepsilon}(x)}\left(s+w_{0}\right) f(s)=\int_{-w_{0}}^{F(\bar{s})}\left(s+w_{0}\right) f(s) .
$$

On the other hand,

$$
\frac{\partial}{\partial t}\left(\mathcal{B}_{\varepsilon}\left(u^{\varepsilon}, x, t\right)\right)=v^{\varepsilon} f_{\varepsilon}\left(u^{\varepsilon}\right) u_{t}^{\varepsilon} \leqslant 0
$$

Therefore,

$$
\mathcal{B}_{\varepsilon}\left(u^{\varepsilon}, x, t\right) \leqslant \int_{-w_{0}}^{F(\bar{s})}\left(s+w_{0}\right) f(s) \quad \text { for } x \text { in } K, t>0 .
$$

As in the proof of Theorem 4.1 we see that, since $u_{t}^{\varepsilon} \leqslant 0$, it holds that $\mathcal{B}_{\varepsilon}\left(u^{\varepsilon}, x, t\right) \rightarrow \bar{M}(x, t)$ in $L_{\mathrm{loc}}^{1}\left(\{u \equiv 0\}^{\circ}\right)$ and, for almost every $(x, t)$, we either have $\bar{M}(x, t)=0$ or $\bar{M}(x, t)=M=$ $\int_{-w_{0}}^{1}\left(s+w_{0}\right) f(s)$. Since

$$
\nabla\left(B_{\varepsilon}\left(u^{\varepsilon}, x, t\right)\right)=v^{\varepsilon} f_{\varepsilon}\left(u^{\varepsilon}\right) \nabla u^{\varepsilon} \rightarrow 0 \quad \text { in } L_{\mathrm{loc}}^{1}\left(\{u \equiv 0\}^{\circ}\right),
$$

it holds that $\bar{M}(x, t)=\bar{M}(t)$ in $\{u \equiv 0\}^{\circ}$. Therefore,

$$
\bar{M}(t) \leqslant \int_{-w_{0}}^{F(\bar{s})}\left(s+w_{0}\right) f(s) \quad \text { a.e. }\{u \equiv 0\}^{\circ} .
$$

Since $F(\bar{s})<1$, it holds that $\bar{M}(t) \equiv 0$.

Thus, for every sequence $\varepsilon_{k} \rightarrow 0$,

$$
\int_{-w_{0}}^{\frac{u^{\varepsilon_{k}}}{\varepsilon_{k}}}\left(s+w_{0}\right) f(s) \rightarrow 0 \quad \text { a.e. }\{u \equiv 0\}^{\circ}
$$

and we deduce that $u^{\varepsilon_{k}}$ satisfies (4.3).

Combining the regularity results for viscosity solutions of [8], Corollary 4.1 and Corollary 4.2, we have the following regularity result for limit functions.

COROLLARY 4.4 Let $u$ be as in Corollary 4.1 or Corollary 4.2. If, moreover, the free boundary $\mathcal{D} \cap \partial\{u>0\}$ is given by $x_{1}=g\left(x^{\prime}, t\right)$ with $g$ Lipschitz continuous, then $u$ is a classical solution of the free-boundary problem (1.4). 


\section{Acknowledgements}

J. F. Bonder is grateful to J. D. Rossi for his constant support and several interesting discussions. This work was partially supported by Universidad de Buenos Aires under grant TX47, by ANPCyT PICT No. 03-00000-00137 and CONICET PIP0660/98. N. I. Wolanski is a member of CONICET.

\section{REFERENCES}

1. Athanasopoulos, I., Caffarelli, L. A., \& Salsa, S. Caloric functions in Lipschitz domains and the regularity of solutions to phase transitions problems. Ann. Math. 143, (1996) 413-434.

2. Berestycki, H., Caffarelli, L. A., \& Nirenberg, L. Uniform estimates for regularization of free boundary problems. In: SADOSKY, C. (ed), Annal. and Partial Diff. Eq., Lecture Notes in Pure and Applied Mathematics, 122. Marcel Dekker, (1988).

3. Buckmaster, J. D. \& Ludford, G. S. S. Theory of laminar flames. Cambridge University Press, Cambridge (1982).

4. CAffarelli, L. A. Uniform Lipschitz regularity of a singular perturbation problem. Diff. and Int. Eq. 8, (1995) 1585-1590.

5. Caffarelli, L. A., Lederman, C., \& Wolanski, N. Uniform estimates and limits for a two phase parabolic singular perturbation problem. Indiana Univ. Math. J. 46, (1997) 453-490.

6. Caffarelli, L. A., Lederman, C., \& Wolanski, N. Pointwise and viscosity solutions for the limit of a two phase parabolic singular perturbation problem. Indiana Univ. Math. J. 46, (1997) 719-740.

7. CAffarelli, L. A. \& VÁzQuez, J. L. A free boundary problem for the heat equation arising in flame propagation. Trans Am. Math. Soc. 347, (1995) 411-441.

8. FORNARI, L. Regularity of the solution and of the free boundary for free boundary problems arising in combustion theory. Preprint.

9. Ladyzhenskaya, O. A., Solonnikov, V. A., \& Ural'tseva, N. N. Linear and quasilinear equations of parabolic type. Transl. Math. Monographs, vol. 23. American Mathematical Society, Providence R.I., (1968).

10. Lederman, C., VAZqueZ, J. L., \& WOLANSKI, N. Uniqueness of solution in a free boundary problem from combustion. Trans AMS. to appear.

11. Lederman, C., Vazquez, J. L., \& Wolanski, N. Uniqueness of solution to a two phase free boundary problem. Preprint.

12. Lederman, C. \& WOLANSKI, N. Viscosity solutions and regularity of the free boundary for the limit of an elliptic two phase singular perturbation problem. Analli della Scuola Normale Sup. Pisa. Ser. IV, XXVII, (1998) 253-288. Fasc. 2

13. Zeldovich, YA. B. \& FRANK-KAMEnEtSKi, D. A. The theory of thermal propagation of flames. $Z h$. Fiz. Khim. 12, (1938) 100-105. (in Russian); English translation in Collected Works of Ya.B. Zeldovich, vol. 1. Princeton Univ. Press, (1992). 